JOURNAL OF THE

AMERICAN MATHEMATICAL SOCIETY

Volume 10, Number 1, January 1997, Pages 1-35

S 0894-0347(97)00195-1

\title{
UNIFORMITY OF RATIONAL POINTS
}

\author{
LUCIA CAPORASO, JOE HARRIS, AND BARRY MAZUR
}

\section{CONTEnTs}

1. Uniformity and correlation 1

1.1. Introduction 1

1.2. Proof of the Uniform Bound Theorem 5

1.3. Proof of the Universal Generic Bound Theorem 8

1.4. The Correlation Theorem 10

2. Examples 11

2.1. Pencils of plane quartics 11

2.2. An isotrivial family 13

2.3. Adjoint conditions and canonical singularities 14

2.4. Proof of Correlation for isotrivial families $\quad 16$

3. Families with maximal variation of moduli 17

3.1. Preliminaries 17

3.2. Proof of Correlation for families with maximal variation 18

4. Families of stable curves with group action 20

4.1. Statement of the main Proposition 20

4.2. Singularities of quotients: adjoint conditions 21

4.3. Proof of the main Proposition and other corollaries 24

5. The general case $\quad 26$

5.1. Stable reduction over arbitrary bases 26

5.2. Completion of the proof of the Correlation Theorem $1.3 \quad 27$

6. The higher-dimensional case 30

6.1. Correlation for families of higher-dimensional varieties 30

6.2. Geometric consequences of the higher-dimensional conjecture. 31

6.3. An arithmetic consequence of the higher-dimensional conjecture 33

References

\section{UNIFORMITY AND CORRELATION}

1.1. Introduction. Let $K$ be a number field. Faltings has proved the finiteness of the number of $K$-rational points on any given curve of geometric genus 2 or more, and his proof gives an effective upper bound for that number. In this paper, we raise the question of how such points behave in families. Specifically, given a family $f: X \rightarrow B$ of curves defined over a number field $K$, how does the set of $K$-rational

Received by the editors September 15, 1994 and, in revised form, March 23, 1995.

1991 Mathematics Subject Classification. Primary 14G05, 14H10.

(C)1997 American Mathematical Society 
points of the fibers vary with $b \in B$, and in particular how does its cardinality behave as a function of $b$ ? For example, is it a bounded function of $b$ ? Given the existence of algebraic families including among their fibers every isomorphism class of smooth curve of genus $g$ over $K$, this is equivalent to the

Uniformity Conjecture. Let $K$ be a number field and $g \geq 2$ an integer. There exists a number $\mathrm{B}(K, g)$ such that for any smooth curve $X$ of genus $g$ defined over $K$,

$$
|X(K)| \leq \mathrm{B}(K, g)
$$

The principal arithmetic results of this paper say that this conjecture, and similar (and even stronger) statements, follow from various purely qualitative conjectures made by Lang and others about the distribution of rational points on higher-dimensional varieties.

Before we state our results, however, we need to introduce briefly the precise forms of the Lang conjectures that we will be invoking.

1.1.1. Lang conjectures. The Lang conjectures extend the Mordell Conjecture, proved by Faltings, to varieties of higher dimension. We will discuss here only a small subset of the various conjectures made by Lang and others; for a more complete discussion see for example [L] and [Vo].

One natural generalization to higher dimensions of the notion of "curve of geometric genus $g \geq 2$ " is "variety of general type". ${ }^{1}$ Of course, if we adopt this as our hypothesis on the variety $X$, we cannot expect the conclusion to be that the set $X(K)$ is finite: a variety of general type may contain rational subvarieties defined over $K$ - a smooth hypersurface of any degree, for example, may contain lines which may in turn have infinitely many rational points. We are thus led to

Conjecture A (Weak Lang Conjecture). If $X$ is a variety of general type defined over a number field $K$, then the set $X(K)$ of $K$-rational points of $X$ is not Zariski dense.

Now, another way to generalize the Mordell Conjecture would be to insist that the conclusion be that $X(K)$ is finite and ask what hypothesis on $X$ would be necessary to imply this. As is suggested by the discussion preceding the Weak Lang Conjecture, it is not enough to ask that $X$ be of general type; we have to ask as well that it not contain subvarieties with too many rational points. Thus, following Lang, we may formulate a conjecture to the effect that if $X$ is any variety defined over a number field $K$ such that $X$ itself and all irreducible, positive-dimensional subvarieties of $X$ are of general type, then $X(K)$ is finite. Note that this conjecture is much less robust than Conjecture A - its hypothesis and conclusion are not birational invariants; and while it is often possible to tell whether a given variety is of general type (for example, any smooth hypersurface of degree $d \geq n+2$ in $\mathbb{P}^{n}$ is of general type), it is difficult to tell whether a given variety satisfies this stronger hypothesis. (Although it is known that hypersurfaces of degree $d \geq 2 n$ in $\mathbb{P}^{n}$ of sufficiently general moduli do satisfy it ([E]), there is no algorithm for determining if a given hypersurface does, and in particular there are no hypersurfaces of any

\footnotetext{
${ }^{1}$ In this paper, the phrase "variety of general type" will only refer to positive-dimensional, irreducible varieties. A smooth projective variety is of general type if and only if it has enough regular pluricanonical forms to effect an embedding of a dense Zariski open subset into projective space; more generally, we say that an arbitrary projective variety is of general type if a desingularization is.
} 
degree in any projective space $\mathbb{P}^{n}$ for $n \geq 3$ that are known to satisfy it.) In any event, since the main geometric results of this paper are birational in nature, we will not be able to use it.

There is, however, a conjecture that combines the two, a sort of fiber product of them. This is what we call the Strong Lang Conjecture:

Conjecture B (Strong Lang Conjecture). Let $X$ be any variety of general type, defined over a number field $K$. There exists a proper closed subvariety $\Xi \subset X$ such that for any number field $L$ containing $K$, the set of $L$-rational points of $X$ lying outside of $\Xi$ is finite.

It is worth remarking that this is actually implied by Conjecture A together with the purely geometric conjecture:

Geometric Lang Conjecture. If $X$ is any variety of general type, the union of all irreducible, positive-dimensional subvarieties of $X$ not of general type is a proper, closed subvariety $\Xi \subset X$.

The Strong Lang Conjecture is known to hold for arbitrary subvarieties of abelian varieties $([\mathrm{F}])$. The Geometric Lang Conjecture has been proved for all surfaces with $c_{1}^{2}>c_{2}([\mathrm{~B}])$, and has recently been announced for all surfaces ([LM]).

1.1.2. The main arithmetic results of this paper. The first statement we will prove is simply that the Weak Lang Conjecture implies the Uniformity Conjecture; that is, the

Theorem 1.1 (Uniform Bound). If Conjecture $A$ is true, then for every number field $K$ and integer $g \geq 2$ there exists an integer $\mathrm{B}(K, g)$ such that no smooth curve defined over $K$ has more than $\mathrm{B}(K, g)$ rational points.

We can deduce much more if we assume the stronger Conjecture B: remarkably, we can say that the number $\mathrm{B}(g, K)$ depends only on $g$ and not on $K !-$ allowing, as we must, a finite list of exceptions for each $K$. Precisely, we will prove

Theorem 1.2 (Universal Generic Bound). The Strong Lang Conjecture implies that for any $g \geq 2$, there exists an integer $\mathrm{N}(g)$ such that for any number field $K$ there are only finitely many smooth curves of genus $g$ defined over $K$ with more than $\mathrm{N}(g)$ K-rational points.

Note that the presence of the finite set of exceptions to the inequality $|X(K)| \leq$ $\mathrm{N}(g)$ is necessary: we can take any given curve $X$ and give it as many $K$-rational points as we like simply by enlarging the number field $K$. We can similarly do this for any finite set of curves $X_{1}, \ldots, X_{n}$ simultaneously, so that the set of exceptions will necessarily grow as $K$ does. What the existence of a universal generic bound $\mathrm{N}(g)$ is saying, though, is that even though we can make up towers of number fields

$$
K_{1} \subset K_{2} \subset K_{3} \subset \ldots
$$

such that $X\left(K_{i}\right) \rightarrow \infty$, any such tower is specific to $X$ in the sense that for each $i$ all but finitely many other curves will continue to have $\mathrm{N}(g)$ or fewer $K_{i}$-rational points.

The proofs of Theorems 1.1 and 1.2 are based on our main geometric statement, the Correlation Theorem 1.3. This will be the subject of 1.1 .3 below. 
Comments. 1. There is a preliminary draft of this paper, [CHM1], available by anonymous ftp from math.harvard.edu. This draft may be more accessible than the present paper to people whose primary background is not in algebraic geometry, in that it has a self-contained treatment of certain aspects of the paper, with more examples.

2. We originally proved something weaker than Theorem 1.2, and we are thankful to Dan Abramovich for providing us with the argument that yields the above statement. For a more specific account of this, see below.

3. Given the existence of bounds such as $\mathrm{B}(K, g)$ and $\mathrm{N}(g)$, it is natural to ask how large they must be. Indeed, we would like to have more numerical examples than we presently have of, for example, curves with given genus and respectably large numbers of $\mathbb{Q}$-rational points. The current record, as far as we know, for curves of genus 2 and 3 is held by A. Brumer, who has an example of a curve of genus 2 with at least $144 \mathbb{Q}$-rational points, and of genus 3 with at least $72 \mathbb{Q}$ rational points. As for the current knowledge concerning the asymptotics of $\mathrm{B}(K, g)$ for fixed $K$ and varying $g$, and of $\mathrm{N}(g)$, see the discussion in [CHM]. Briefly, the best estimates to date are $\mathrm{B}(\mathbb{Q}, g) \geq 8 \cdot g+12$ (due to Brumer) and $g b \geq 16(g+1)$ (coming from different constructions, one due to Brumer and another to Mestre). We also thank David Eisenbud, Noam Elkies and Nick Shepherd-Barron for help in making contructions that guarantee some present-day record-holding lower bounds in particular instances, such as $\mathrm{N}(2) \geq 128$ and $\mathrm{N}(3) \geq 72$.

4. The result of Theorem 1.1 has been generalized in two ways by Dan Abramovich. In [Ab1] he proves an analogous result for stably integral points on elliptic curves. In $[\mathrm{Ab} 2]$ he strengthens the statement of Theorem 1.1 substantially, showing that, if we assume only the Weak Lang Conjecture, then the bound $\mathrm{B}(K, g)$ of the theorem remains bounded as $K$ varies over all quadratic extensions of a given number field.

1.1.3. The main geometric results of this paper: Correlation. To prove Theorems 1.1 and 1.2 we need to study a phenomenon that occurs in families of curves of genus at least 2, and which we call Correlation or n-point Correlation. To define this concept in fuller generality, let

$$
f: X \longrightarrow B
$$

be a proper morphism of integral ${ }^{2}$ varieties over $K$, such that the generic fiber of $f$ over $B$ is a smooth variety of general type. As is customary, we will denote the fiber of $f$ over any point $b$ in $B$ as $X_{b}$; that is, $X_{b}:=f^{-1}(b)$. For $n \geq 0$ we denote by $X_{B}^{n}$ the unique irreducible component of the $n^{\text {th }}$ fiber product of $X$ over $B$, which dominates $B$ (and we set $X_{B}^{0}=B$ ), so that we get a natural morphism

$$
f_{n}: X_{B}^{n} \longrightarrow B \text {. }
$$

We say that the family $f: X \longrightarrow B$ has correlation if there is an integer $n$ such that $X_{B}^{n}$ admits a rational dominant map, defined over $K$, to a variety of general type. The question is: do all such families have correlation?

We have adopted the term "correlation" in anticipation of using Lang's conjecture, and here is why. Let us consider the case in which our $X \rightarrow B$ is a family of curves of genus at least 2. Faltings' theorem guarantees that the $K$-rational points of each fiber of $X$ over $B$ are finite in number. But, of course, we have no idea

${ }^{2}$ By "integral" variety we will mean reduced, irreducible scheme. 
of how they are distributed in $X$, unless $X$ itself is a variety of general type; for example, they may very well form a dense subset, if $X$ is rational. In other words, there is no evident "correlation" or contiguity between the (finite) set of rational points of one fiber and another. But suppose, say, that the symmetric square $X_{B}^{2}$ dominates a variety of general type. Then, by Lang's conjecture, the $K$-rational points of $X_{B}^{2}$ lie in a proper, closed subvariety of $X_{B}^{2}$; that is to say, there are "algebraic function relations" that govern the placement of couples of $K$-rational points in a fiber. These "algebraic function relations" we think of as being like statistical "two-point correlation" among $K$-rational points of fibers. Similarly, if $X_{B}^{n}$ dominates a variety of general type, Lang's conjecture would imply the existence of the analogue of an " $n$-point correlation" among $K$-rational points of fibers.

In this paper, we will show that every family of generically smooth curves of general type has correlation. This will be the key ingredient in the proofs of Theorems 1.1 and 1.2. Here is the precise statement:

Theorem 1.3 (Correlation). Let $f: X \longrightarrow B$ be a proper morphism of integral varieties, whose general fiber is a smooth curve of genus at least 2 .

Then for $n$ sufficiently large, $X_{B}^{n}$ admits a dominant rational map $h$ to a variety of general type $W$. Moreover, if $X$ is defined over the number field $K$, then $W$ and $h$ are defined over $K$.

That is, using our terminology, such a family $f: X \longrightarrow B$ has Correlation.

We are thankful to János Kollár and Eckhart Viehweg for conversations concerning the case of families with higher-dimensional fibers. See Section 6 for an account of this, and for an account of some of the arithmetic applications (which are even more sweeping uniformity statements than those discussed in the preceding section).

Addendum. A few more words about the variety $W$ are in order. Recall that a family of generically smooth curves $f: X \rightarrow B$, such as the one in the Theorem, yields a canonical rational morphism $\phi: B \rightarrow M_{g}$ to the moduli space of smooth curves $M_{g}$ (cf. Section 3 for details). Up to restricting to a dense subset of $B$, so that all maps will be regular and $f$ a family of smooth curves, we will show that there is a commutative diagram of morphisms

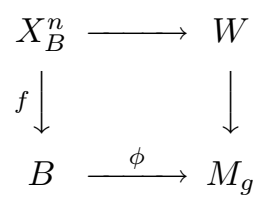

and that $W$ is the quotient by a finite group of a tautological family, over a finite cover $\Sigma$ of $M_{g}$.

1.2. Proof of the Uniform Bound Theorem. We are now going to prove Theorem 1.1, assuming the Weak Lang Conjecture and Theorem 1.3.

Throughout this section we will consider a family $f: X \longrightarrow B$ of generically smooth curves of genus $g>1$ as in Theorem 1.3.

Lemma 1.1. Let $f: X \longrightarrow B$ be a family of curves as above. Then there exist a non-empty Zariski-open subset $U_{0}$ of $B$ and an integer $\mathrm{N}$ such that for every $K$ rational point $b \in U_{0}$ the number of $K$-rational points of the fiber $X_{b}$ is at most equal to $\mathrm{N}$. 
Proof. Throughout this proof, all morphisms and varieties will be defined over $K$, and we will not specify that any further.

By Theorem 1.3, our family has correlation, hence we can consider an integer $n$ such that $X_{B}^{n}$ dominates a variety of general type $W$, via a rational map $h$. By Conjecture A, there is an open subset $W^{\prime}$ of $W$ containing no $K$-rational points. Letting $U \subset X_{B}^{n}$ be the largest open subset over which the morphism to $W$ is regular and such that $g(U) \subset W^{\prime}$, we see that $U$ contains no $K$-rational points, and therefore there is a proper closed subvariety $Z$ of $X_{B}^{n}$ containing all the $K$ rational points of $X_{B}^{n}$. Let $j$ be an integer such that $1 \leq j \leq n$, and denote by $\pi_{j}$ the morphism corresponding to the natural family of curves $\pi_{j}: X_{B}^{j} \rightarrow X_{B}^{j-1}$ obtained by forgetting the last coordinate. Clearly, $\pi_{1}=f$.

It is useful to think of the proof of our Lemma as falling into two parts.

Part 1 . We fix a closed subvariety $Z_{n} \subset X_{B}^{n}$, containing all $K$-rational points of $X_{B}^{n}$, and we let $U_{n}$ be its complement; that is, $U_{n}:=X_{B}^{n} \backslash Z_{n}$. Then we let $Z_{n-1}$ be the largest closed subvariety of $X_{B}^{n-1}$ such that the full inverse image of it in $X_{B}^{n}$ is contained in $Z_{n}$; that is, $\pi_{n}^{-1}\left(Z_{n-1}\right) \subset Z_{n}$. Then we proceed inductively to define $Z_{j}$ to be the largest closed subvariety of $X_{B}^{j}$ such that its inverse image via $\pi_{j+1}$ is contained in $Z_{j+1}$. Another (equivalent) way to define $Z_{j}$ is as the largest closed subvariety of $X_{B}^{j}$ such that its inverse image in $X_{B}^{n}$ is entirely contained in $Z$. Notice that either $Z_{j}$ is fibered in curves over $Z_{j-1}$, or $Z_{j-1}$ is empty. Let

$$
U_{j}:=X_{B}^{j} \backslash Z_{j}
$$

The variety $U_{j}$ is clearly open, and, by construction, the restriction of the projection $\pi_{j}$ to $\pi_{j}^{-1}\left(U_{j-1}\right) \cap Z_{j}$ is finite over its image in $U_{j-1} \subset X_{B}^{j-1}$. Now, $Z_{j}$ may be reducible, so let us index its irreducible components by $\alpha$ so that $Z_{j}=\bigcup_{\alpha}\left(Z_{j}\right)^{(\alpha)}$. Then we let $d_{j}$ be the sum over $\alpha$ of the degrees of $\pi_{j}$ restricted to $\left(Z_{j}\right)^{(\alpha)}$; that is,

$$
d_{j}:=\sum_{\alpha}\left(\operatorname{deg}\left\{\pi_{j}: \pi_{j}^{-1}\left(U_{j-1}\right) \cap\left(Z_{j}\right)^{(\alpha)} \longrightarrow U_{j-1}\right\}\right) .
$$

The point is, $d_{j}$ is an upper bound on the cardinality of the fibers of $Z_{j}$ over $U_{j-1}$.

Our goal is to show that we can take the following as the upper bound on the number of $K$-rational points of the fibers over $U_{0}$ :

$$
\mathrm{N}=\max \left\{d_{j}, \quad 0<j \leq n\right\} .
$$

Part 2. Now we consider $U_{0} \subset B$. Let $b$ be a $K$-rational point of $U_{0}$, and let $j(b)$ denote the smallest integer $j$ such that all $K$-rational points of $X_{B}^{j}$ lying over $b$ are contained in $Z_{j}$. Since $U_{n}$ contains no $K$-rational points, we have that $j(b) \leq n$ for every $b$ in $U_{0}(K)$.

Equivalently, $j(b)$ is the largest integer for which there exists a $K$-rational point $u$ of $U_{j-1}$ such that $f_{j-1}(u)=b$.

Fix such a $u$, and set $j=j(b)$ for $b=f_{j-1}(u)$. We claim that the fiber of $X$ over $b$ has at most $d_{j} K$-rational points. To see this, denote by $X_{u}$ the fiber of $\pi_{j}: X_{B}^{j} \rightarrow X_{B}^{j-1}$ over the point $u$, so that $X_{u}$ is isomorphic to $X_{b}$ over $K$. By our choice of $j, U_{j}$ contains no $K$-rational point above $u$, which is to say that the set of $K$-rational points of $X_{u}$ is entirely contained in $Z_{j}$. Hence we conclude that

$$
\left|X_{b}(K)\right|=\left|X_{u}(K)\right| \leq d_{j},
$$

which proves our claim. 
Therefore, the open subvariety $U_{0}$ of $B$ and the integer $\mathrm{N}=\max \left\{d_{j}, 0<j \leq n\right\}$ have the properties asserted in our statement.

Proof of Theorem 1.1. To conclude the proof of the Theorem, we would like to have a family (a finite collection of families would be enough, in fact) with the property of being "global" in the following sense: we require that every smooth curve of genus $g$, defined over $K$, appears at least once as a fiber of our family (or collection of families). Precisely, the fact that we need to extract and use is the following

Fact: Existence of Global Families of Curves. For any integer $g \geq 0$ and any number field $K$, there exists a global family for curves of genus $g$, that is, there exists a proper, flat $K$-morphism of integral, projective varieties (defined over $K$ ) $\bar{f}: \bar{X} \longrightarrow \bar{B}$ such that given any curve $C$ of genus $g$ defined over $K$ there exists a point $b \in \bar{B}(K)$ such that the fiber of $\bar{X}$ over $b$ is isomorphic to $C$.

To establish this, we will invoke the existence of the Hilbert scheme. The Hilbert scheme $\mathrm{Hilb}_{r}^{\mathrm{h}(t)}$ is the fine moduli scheme for closed subschemes of a fixed projective space $\mathbb{P}^{r}$ with fixed Hilbert polynomial $\mathrm{h}(t)$. In particular, the $K$-rational points of $\mathrm{Hilb}_{r}^{\mathrm{h}(t)}$ will be in one-to-one correspondence with closed subschemes of $\mathbb{P}^{r}$ defined over $K$ having $\mathrm{h}(t)$ as Hilbert polynomial; and the fact that $\operatorname{Hilb}_{r}^{\mathrm{h}(t)}$ is a fine moduli scheme translates into the existence of a universal family $\mathcal{X}_{r}^{\mathrm{h}(t)}$ over it:

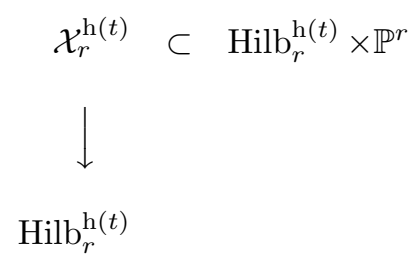

For our purposes, we may consider that Hilbert scheme whose numerical invariants are such that it parametrizes (among many other things) all $n$-canonical embeddings of all curves of genus $g \geq 2$, with $n$ any fixed integer greater than 2 . That is to say that we pick the following numerical data: take $n=10$ say, and $d=10 \cdot(2 g-2)$; the Hilbert polynomial will then be $\mathrm{h}(t)=d \cdot t-g+1$ and the projective space will have dimension $r=d-g$.

It is well known (cf. [GIT]) that the locus of pluricanonical smooth curves in $\operatorname{Hilb}_{r}^{\mathrm{h}(t)}$ is a locally closed, irreducible, smooth subscheme of the Hilbert scheme; we will denote it by $H_{g}$ :

$$
\begin{aligned}
H_{g}:=\left\{h \in \operatorname{Hilb}_{r}^{\mathrm{h}(t)}:\right. & X_{h} \text { is smooth, connected, } \\
& \text { non-degenerate and } \left.\mathcal{O}_{X_{h}}(1) \cong K_{X_{h}}^{\otimes 10}\right\} .
\end{aligned}
$$

We can take our global family $\bar{f}: \bar{X} \longrightarrow \bar{B}$ to be the closure of $H_{g}$ in $\operatorname{Hilb}_{r}^{\mathrm{h}(t)}$ with the restriction of the universal family.

We conclude the proof of the Theorem by a downwards induction argument on the dimensions of the bases of our families. We start with a global family, $\bar{f}: \bar{X} \longrightarrow \bar{B}$ as described above. The previous Lemma produces a finite upper bound $\mathrm{N}_{0}$ on the number of rational points for the fibers of our global family, except for the fibers lying over a lower dimensional subvariety $\overline{B_{1}}$ of our base $\bar{B}$. Let $B_{1}$ be the union of all the irreducible components of $\overline{B_{1}}$, which are not contained in the discriminant locus of the original family; that is, $B_{1}$ is the union of those irreducible 
components whose general fiber is a smooth curve. Let $X_{1}$ be the restriction of the original family to $B_{1}$; this will be a new family of curves whose base $B_{1}$ will be the union of a finite number of lower dimensional closed subvarieties of $\bar{B}$. We can then apply the Lemma to these families, and obtain an upper bound $\mathrm{N}_{1}$ on the number of $K$-rational points of the fibers over an open and dense subset of $B_{1}$. We can iterate this process, and at each application of our Lemma we get an upper bound $\mathrm{N}_{i}$ for the $K$-rational points lying over the fibers of an open dense subset of $B_{i}$. Since of course $\operatorname{dim} B_{i}<\operatorname{dim} B_{i-1}$ we will arrive at an end in finitely many steps. Therefore if we take $\mathrm{B}(K, g)$ equal to the maximum of the $\mathrm{N}_{i}$, we have that every fiber over $\bar{B}$ has at most $\mathrm{B}(K, g) K$-rational points. Since such a family was global, such a $\mathrm{B}(K, g)$ satisfies the requirement of the Theorem.

1.3. Proof of the Universal Generic Bound Theorem. Now, assuming the Strong Lang Conjecture together with Theorem 1.3, we will prove Theorem 1.2.

The proof is very similar to that of Theorem 1.1. It consists in two steps. In the first step - which is basically the analogue of the Lemma in the proof of Theorem 1.1, we find a universal bound on the number of rational points of the fibers over an open dense subset of the base of our family. The second step sets up an iterating procedure in finitely many steps, starting from a global family. First of all, let us revisit the construction of the proof of the Lemma. Recall that we had the following set up: $n$ is an integer such that $X_{B}^{n}$ dominates a variety of general type $W$ via a map $h$; after restricting to an appropriate dense subvariety of $B$, we can assume that $h$ is a regular morphism, without really changing the format of the proof. We reproduce here the diagram given by Theorem 1.3:

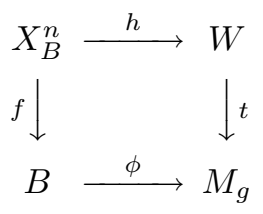

We obtained bounds on the number of $K$-rational points of the fibers of $f$, defined over $K$. Now we will study how these bounds depend on the number field $K$.

By the Strong Lang Conjecture, there exists a proper, closed subvariety $\Xi$ of $W$ such that for any number field $K$ the set $W(K)$ is contained in the union of $\Xi$ and a finite subset $E_{K}$ of $W$.

Let $\mathcal{E}_{g, K}(f)$ be the image of $E_{K}$ in $M_{g}$ via the map $t$, and let $B^{\prime}$ be the complement in $B$ of the preimage of $\mathcal{E}_{g, K}(f)$ via $\phi$; that is,

$$
B^{\prime}:=B \backslash \phi^{-1}\left(\mathcal{E}_{g, K}(f)\right) .
$$

At this point, let us restrict our family to the base $B^{\prime}$, but to keep our notation from being too heavy, we relabel $B^{\prime}$ as $B$. Having done this, let $Z \subset X_{B}^{n}$ be the inverse image of $\Xi$ in $X_{B}^{n}$ under $h$, so that, for any number field $K, Z$ contains all the $K$-rational points of $X_{B}^{n}$. We now make the construction of Part 1 of the proof of the Lemma, obtaining closed subvarieties $Z_{j} \subset X_{B}^{j}$, their complements $U_{j}$, and the integers $d_{j}$, for $0<j \leq n$. Of course the point is that all of this does not depend on $K$.

Now we pass to Part 2. For each number field $K$ we can proceed as we did for the proof of the Lemma. To start, we associate to each point $b \in U_{0}(K)$ an integer $j_{K}(b)$, playing the same role as $j(b)$ there. 
Specifically, let $j(b)$ denote the smallest integer $j$ such that all $K$-rational points of $X_{B}^{j}$ lying over $b$ are contained in $Z_{j}$; equivalently, $j(b)$ is the largest integer for which there exists a $K$-rational point $u$ of $U_{j-1}$, such that $f_{j-1}(u)=b$. (The only difference now is that we need to consider the dependence of the index $j$ on $K$.) Now Part 2 gives us

$$
\left|X_{b}(K)\right| \leq d_{j_{K}(b)}
$$

where recall that $d_{j}$ was defined to be the sum of the degrees of the finite morphisms obtained by restricting $\pi_{j}$ to the irreducible components of $\pi_{j}^{-1}\left(U_{j-1}\right) \cap Z_{j}$. Hence there is a finite upper bound on the $d_{j_{K}(b)}$ 's, for $K$ and $b$ varying, which does not depend on $K$; in fact we have

$$
\max \left\{d_{j_{K}(b)}, \forall K, \forall b \in U_{0}(K)\right\}=\max \left\{d_{j}, \quad 0<j \leq n\right\}=\mathrm{N} .
$$

We have therefore concluded that there is an integer $\mathrm{N}$ and a dense subset $U_{0}$ of $B$, such that for any $K$, the number of rational points of any fiber over $U_{0}$ is less than $\mathrm{N}$.

The inductive process of considering families with successive lower dimensional bases proceeds exactly as before, by starting with a global family $\bar{f}: \bar{X} \longrightarrow \bar{B}$ such as the one described in Section 1.2. Let us index these families by the index $i \geq 0$, so that the original global family corresponds to $i=0$. We obtain that - except for the exclusion of the inverse images of the finite sets $\mathcal{E}_{g, K}\left(f_{i}\right)$ in $B_{i}$, for each of the families that we must deal with, during our inductive procedure, we get an upper bound $\mathrm{N}_{i}$, independent on the number field $K$, for the number of $K$-rational points that lie on our curves. There being only a finite number of such families, we obtain that the maximum over the $\mathrm{N}_{i}$ is a finite number, which will therefore be our $\mathrm{N}(g)$; moreover, by taking $\mathcal{E}_{g, K}$ to be the union of all the exceptional sets $\mathcal{E}_{g, K}\left(f_{i}\right)$, we have that $\mathcal{E}_{g, K}$ is also finite.

We have thus shown a uniform bound, independent of $K$, on the number of $K$-rational points of any curve $X_{b}$ of our family such that $\varphi(b)$ does not lie in $\mathcal{E}_{g, K}$. This is not quite strong enough to establish the conclusion of the Theorem as stated, however, since there may be infinitely many fibers $X_{b}$ that are isomorphic over the algebraic closure $\overline{\mathbb{Q}}$, and so correspond to the same point $\varphi(b)$ in the moduli space, but that are not isomorphic over $K$. (We had originally stated Theorem 1.2 in this way, and we thank Dan Abramovich for suggesting the present stronger formulation, and for showing us the following argument to prove it.) To conclude the proof, we must show that in this case, for each $K$, there are only finitely many $K$-isomorphism classes of such fibers $X_{b}$ with more than $\mathrm{N}(g)$ points.

For this purpose, we may restrict our attention to an isotrivial family $X \rightarrow B$ defined over $K$. By an isotrivial family we will mean one with an open subset $U \subset B$ such that the fibers $X_{b}$ with $b \in U(\overline{\mathbb{Q}})$ are all isomorphic to a fixed curve $C$ over $\overline{\mathbb{Q}}$. This is equivalent (cf. [SGA]) to the condition that for some generically finite map $B^{\prime} \rightarrow B$ the pullback family $X^{\prime}=X \times{ }_{B} B^{\prime}$ is birational (again, over $\overline{\mathbb{Q}}$ ) to a product $B^{\prime} \times C$. Note that we don't actually have to go all the way to $\overline{\mathbb{Q}}$ : there will exist some finite extension $L_{0}$ of $K$, a curve $C$ defined over $L_{0}$ and a birational isomorphism of $X^{\prime}$ with $B^{\prime} \times C$ is defined over $L_{0}$. One key observation: for any point $b \in B$ and any $b^{\prime} \in B^{\prime}(\overline{\mathbb{Q}})$ lying over $b$, the fiber $X_{b}$ will be isomorphic to $C$ over the compositum of the field $L_{0}$ and the field of definition of $b^{\prime}$.

In this situation, let $Y=X_{B}^{n}$ and let $h: Y \rightarrow W$ be as above the map of $Y$ to a variety $W$ of general type. In fact, as we will establish in Section 2.4, in the case 
of an isotrivial family, we can say very explicitly what $h$ and $W$ look like: we can in this case take $B^{\prime} \rightarrow B$ a Galois cover, with Galois group $G$ a subgroup of the group of automorphisms of $C$, and $W=C^{n} / G$ the quotient of $C^{n}$ by the group $G$ acting diagonally. Setting $W^{\prime}=C^{n}$, we have a diagram

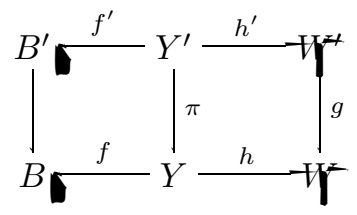

where $Y^{\prime}:=Y \times_{B} B^{\prime}=B^{\prime} \times C^{n}, W^{\prime}:=C^{n}$ and $h^{\prime}$ is the projection map. In particular, $Y^{\prime}$ is the fiber product

$$
Y^{\prime}=W^{\prime} \times_{W} Y
$$

Now suppose that $x$ is any $K$-rational point of $W$, and suppose $b \in B(K)$ is any $K$-rational point such that

$$
Y_{b}(K) \cap h^{-1}(x) \neq \emptyset,
$$

i.e., the fiber $Y_{b}$ contains a $K$-rational point $y$ lying over $x$. We have to show that the fiber $X_{b}$ of our original family belongs to one of finitely many $K$-isomorphism classes.

To see this, let $L_{1}$ be any number field such that there is an $L_{1}$-rational point $x^{\prime} \in W^{\prime}\left(L_{1}\right)$ lying over $x$, and let $L$ be the compositum of $L_{0}$ and $L_{1}$. The crucial point is that we now have an $L_{1}$-rational point $\left(x^{\prime}, y\right) \in W^{\prime} \times_{W} Y=Y^{\prime}$, and hence $B^{\prime}$ contains an $L_{1}$-rational point $b^{\prime}=f^{\prime}\left(x^{\prime}, y\right)$ lying over $b$. From this it follows, as we observed above, that the fiber $X_{b}$ is isomorphic to $C$ over $L$. But now we have achieved our goal, because there are only finitely many isomorphism classes of curves defined over $K$ that become isomorphic over a given finite extension $L$.

In conclusion: each point $x$ of $W$ not contained in the Langian exceptional locus $\Xi$ contributes an extra $K$-rational point only to fibers $X_{b}$ belonging to one of a finite set of isomorphism classes over $K$; the result follows.

1.4. The Correlation Theorem. To conclude this section, we will describe the structure of the remainder of our paper, most of which will be devoted to the proof of our main geometric result, the Correlation Theorem. Our proof of this will occupy Sections 3, 4, and 5 .

To begin with, Section 2 introduces two examples of families of curves, pencils of plane quartics and isotrivial pencils of hyperelliptic curves (we also include a complete proof of the Theorem in the case of an arbitrary isotrivial family). These serve to illustrate the phenomenon that Theorem 1.3 asserts holds in general, and to give a sense of what we might hope to be true in general. They also introduce a notion that will be essential in the sequel: adjoint conditions and canonical singularities (cf. 2.3).

In Section 3 we prove Correlation for complete families of stable curves having maximal variation of moduli (see Section 3 for the terminology used below). This is largely a matter of quoting the relevant sources in the literature, notably Viehweg ([V1]) and Elkik ([E]). Alternatively, for an ad-hoc but self-contained proof, see [CHM1].

In Section 4, we extend this result: we consider again a complete family $f$ : $X \rightarrow B$ of stable curves having maximal variation, but now with a finite group $G$ 
acting on $X$ and $B$. The result we want is that, for $n$ sufficiently large, the quotient $X_{B}^{n} / G$ of the fiber power $X_{B}^{n}$ by $G$ is again of general type. The reason for this is that, in order to reduce to the case of families of stable curves, we have to make base changes; and in order for our result to say something about rational points on the fibers of the original family we need in the end to take quotients. (After all, the family we obtain after base change need not have any fibers defined over our original number field $K$.) The main additional ingredient in the proof is an analysis of the adjoint conditions imposed by the singularities of the quotients; we show in Section 4.2 that these conditions remain bounded as $n$ grows and this allows us to deduce the main result, Proposition 4.1.

In Section 5 we complete the proof of the Correlation Theorem by showing how to reduce the case of an arbitrary family $X \rightarrow B$ to the case already considered. Specifically, we show that, after a base change, we can construct a family $T \rightarrow \Sigma$ of stable curves whose fibers are the same as those of $X$ on an open set, and that after a further base change $B^{\prime} \rightarrow B$ we can express $X^{\prime}=X \times_{B} B^{\prime} \rightarrow B$ as the pullback of the family $T \rightarrow \Sigma$. Moreover, we show that we can make the construction in such a way that the group introduced in making the base change acts as well on the family $T \rightarrow \Sigma$. We then apply the results of Section 4 to the family $T \rightarrow \Sigma$ to deduce the theorem.

Finally, we append in Section 6 some speculations on the case of families of varieties of general type with higher-dimensional fibers. We state an analogue of the Correlation Theorem, discuss possible ingredients of a proof, and mention at least some of the consequences, geometric as well as arithmetic.

\section{EXAMPLes}

The purpose of this section is to discuss the type of difficulties that we will have to overcome in proving the Correlation Theorem. We will do that by directly proving the Theorem for two examples of families of curves.

2.1. Pencils of plane quartics. Let $f(x, y)$ and $g(x, y)$ be general quartic polynomials, and consider the family of plane quartic curves given by

$$
f(x, y)+t \cdot g(x, y)=0
$$

parametrized by a line $B \cong \mathbb{P}^{1}$ with coordinate $t$. Homogenizing the polynomial $f+t \cdot g$ with respect to the variables $t$ and $(x, y)$ separately, the total space of this family can be realized as a hypersurface $X \subset \mathbb{P}^{1} \times \mathbb{P}^{2}$ of bidegree $(1,4)$, smooth as long as the base points of the pencil are distinct. The projection map

$$
\pi_{2}: X \rightarrow \mathbb{P}^{2}
$$

expresses $X$ as the blow-up of $\mathbb{P}^{2}$ at the 16 base points of the pencil; in particular, $X$ is a rational variety over $K$. Note that this means that the rational points of $X$ are everywhere dense, in the classical as well as the Zariski topology. We think of this as suggesting that there are no "relations" between the finite sets of rational points $X_{t}(K)$ as $t$ varies through $K$.

It also follows, of course, that $X$ is not of general type, but we could also see this by calculating its canonical bundle using the adjunction formula. Since the canonical bundle of $\mathbb{P}^{1} \times \mathbb{P}^{2}$ is

$$
K_{\mathbb{P}^{1} \times \mathbb{P}^{2}}=\mathcal{O}_{\mathbb{P}^{1} \times \mathbb{P}^{2}}(-2,-3)
$$


and

we have

$$
\mathcal{O}_{\mathbb{P}^{1} \times \mathbb{P}^{2}}(X) \cong \mathcal{O}_{\mathbb{P}^{1} \times \mathbb{P}^{2}}(1,4)
$$

$$
K_{X}=K_{\mathbb{P}^{1} \times \mathbb{P}^{2}}(X) \otimes \mathcal{O}_{X}=\mathcal{O}_{X}(-1,1)
$$

and no positive power of this line bundle can have regular global forms.

On the other hand, the same calculation allows us to conclude that the fiber square of $X$ over $B$ is of general type: the fiber square $Y=X \times_{B} X$ is given in $\mathbb{P}^{1} \times \mathbb{P}^{2} \times \mathbb{P}^{2}$ as the locus of the (homogenization of the) two equations

$$
f(x, y)+t \cdot g(x, y)=0
$$

and

$$
f(u, v)+t \cdot g(u, v)=0
$$

in other words, it is a complete intersection in $\mathbb{P}^{1} \times \mathbb{P}^{2} \times \mathbb{P}^{2}$ of two hypersurfaces $X_{1}$ and $X_{2}$ of tridegrees $(1,4,0)$ and $(1,0,4)$. Let $\omega_{Y}$ be the dualizing sheaf of $Y$. Applying adjunction once more, we see that

$$
\begin{aligned}
\omega_{Y}= & \omega_{\mathbb{P}^{1} \times \mathbb{P}^{2} \times \mathbb{P}^{2}}\left(X_{1}+X_{2}\right) \otimes \mathcal{O}_{Y} \\
= & \mathcal{O}_{\mathbb{P}^{1} \times \mathbb{P}^{2} \times \mathbb{P}^{2}}(-2,-3,-3) \otimes \mathcal{O}_{\mathbb{P}^{1} \times \mathbb{P}^{2} \times \mathbb{P}^{2}}(1,4,0) \\
& \otimes \mathcal{O}_{\mathbb{P}^{1} \times \mathbb{P}^{2} \times \mathbb{P}^{2}}(1,0,4) \otimes \mathcal{O}_{Y} \\
= & \mathcal{O}_{Y}(0,1,1) .
\end{aligned}
$$

In particular, the dimension of the space of sections of $\left(\omega_{Y}\right)^{\otimes m}=\mathcal{O}_{Y}(0, m, m)$ grows as a cubic polynomial in $m$.

We would like to use this fact to conclude that $Y$ is of general type, i.e., that the tensor powers of $\omega_{\widetilde{Y}}\left(=K_{\widetilde{Y}}\right)$ have enough sections to define an embedding of an open subset of $\widetilde{Y}$ in projective space, for a desingularization $\tilde{Y}$ of $Y$. This is easy to do in the present case by exhibiting a desingularization $\widetilde{Y} \rightarrow Y$ and relating $\omega_{\widetilde{Y}}$ to $\omega_{Y}$. Explicitly, in a neighborhood of each singular point $p$ of a fiber of $X \rightarrow B$ we have local coordinates $z, w$ so that $X$ has local equation $z w-t=0$. The fiber square $Y$ will then have local equation

$$
z w-t=a b-t=0,
$$

i.e., it is the zero locus, in a smooth fourfold $Z$ with local coordinates $(z, w, a, b)$, of the single equation $z w=a b$. This we recognize as an ordinary double point of a threefold, which is resolved by simply blowing up $Z$ at the point $p$ and taking $\tilde{Y}$ as the proper transform of $Y$ in $\widetilde{Z}$. In terms of the exceptional divisor $E$ of the blow-up, we have

$$
\omega_{\widetilde{Z}}=\left(\pi^{*} \omega_{Z}\right)(3 E)
$$

and

$$
\mathcal{O}_{\widetilde{Z}}(\widetilde{Y})=\left(\pi^{*} \mathcal{O}_{Z}(Y)\right)(-2 E)
$$

so we see that the canonical bundle of the resolution is given by

$$
\omega_{\tilde{Y}}=\left(\pi^{*} \omega_{Y}\right)(E)
$$

where $E$ is the exceptional divisor; in particular, we see that the singularities of $Y$ are canonical (for the definition of canonical singularities see Section 2.3, where we treat this problem in greater generality). It follows that regular sections of tensor powers of $\omega_{Y}$ pull back to regular sections of powers of $\omega_{\widetilde{Y}}$, and we conclude that $Y$ is of general type. 
2.2. An isotrivial family. Consider in this section a family $f: X \rightarrow B$ that is isotrivial in the sense that the fibers $\left\{X_{b}: b \in U\right\}$ lying over an open subset $U \subset B$ are all isomorphic, over a large enough field $K$ to a fixed curve $C$. In this case we cannot expect the spaces $X_{B}^{n}$ to be of general type - as we will see below, they are finite quotients of products $B_{1} \times C^{n}$, where $B_{1}$ will be a suitably defined base change of $B$ - but they do admit dominant rational maps to lower-dimensional varieties of general type.

Let us begin with a concrete example. We fix a polynomial

$$
\begin{aligned}
f(x) & =x^{n}+a_{n-1} x^{n-1}+\ldots+a_{0} \\
& =\left(x-\alpha_{1}\right) \cdot\left(x-\alpha_{2}\right) \cdot \ldots \cdot\left(x-\alpha_{n}\right)
\end{aligned}
$$

of degree $n=2 g+2$ with no multiple roots, $a_{i} \in K$, and consider the isotrivial family $X \rightarrow \mathbb{A}^{1}$ of hyperelliptic curves of genus $g$, parametrized by the affine line with coordinate $t$, given by

$$
t \cdot y^{2}=f(x)
$$

We view this family as a pencil of curves of bidegree $(2 g+2,2)$ on $\mathbb{P}^{1} \times \mathbb{P}^{1}$, so that its total space is a blow-up of $\mathbb{P}^{1} \times \mathbb{P}^{1}$; in particular, is rational.

When we take the fiber square $X_{B}^{2}$ of $X$ over $\mathbb{A}^{1}=B$, however, we see something different: the total space of $X_{B}^{2}$ has equations

$$
t \cdot y^{2}=f(x), \quad t \cdot v^{2}=f(u)
$$

so that $X_{B}^{2}$ is birational to the threefold

$$
\left\{(x, u, v, y): v^{2} \cdot f(x)=y^{2} \cdot f(u)\right\} \subset \mathbb{A}^{4} .
$$

In particular, it maps to the surface $V$ given by

$$
\left\{(x, u, w): w^{2}=f(u) \cdot f(x)\right\}
$$

by the map sending $(x, u, v, y)$ to $(x, u, v \cdot f(x) / y)$.

Now, the surface $V$ is birationally the double cover of $\mathbb{P}^{1} \times \mathbb{P}^{1}$ branched along the locus $f(x) \cdot f(u)=0$ - that is, the union of $2 g+2$ fibers from each ruling of $\mathbb{P}^{1} \times \mathbb{P}^{1}$. As such, it is not hard to see that $V$ is a surface of general type. (It should be observed that $V$ is not a smooth surface, but is singular at the $(2 g+2)^{2}$ points $\left(\alpha_{i}, \alpha_{j}, 0\right)$ lying over the double points of the branch divisor.) For example, we can simply write down the canonical differentials on $V$ : if we set

$$
\theta=\frac{d u \wedge d x}{w}
$$

we can readily check that the products

$$
\theta_{k, l}=u^{k} \cdot x^{\ell} \cdot \theta
$$

give regular canonical differentials on the smooth locus of $V$ for all $0 \leq k, \ell \leq g-1$. Alternatively (equivalently), we can apply the Riemann-Hurwitz formula to the cover $\eta: V \rightarrow \mathbb{P}^{1} \times \mathbb{P}^{1}$ : the ramification divisor $R$ of $\eta$ is the zero-divisor of the function $w$ on $V$, and from the equality

$$
2 R=\left(w^{2}\right)=(f(u))+(f(x))
$$

we may deduce that, on the smooth locus of $V$,

$$
\begin{aligned}
K_{V} & =\pi^{*}\left(K_{\mathbb{P}^{1} \times \mathbb{P}^{1}}\right) \otimes \mathcal{O}_{V}(R) \\
& =\pi^{*}\left(K_{\mathbb{P}^{1} \times \mathbb{P}^{1}} \otimes \mathcal{O}_{\mathbb{P}^{1} \times \mathbb{P}^{1}}(g+1, g+1)\right)=\pi^{*}\left(\mathcal{O}_{\mathbb{P}^{1} \times \mathbb{P}^{1}}(g-1, g-1)\right)
\end{aligned}
$$

which is ample. 
Finally, we need to deal with the singular points of $V$. In fact, these do not present a difficulty in this case: at the singular point $\left(\alpha_{i}, \alpha_{j}, 0\right)$, after replacing $u$ and $x$ by $\left(u-\alpha_{i}\right)$ and $\left(x-\alpha_{j}\right), V$ will have local equation

$$
w^{2}=u \cdot x
$$

and blowing up once we arrive at a smooth surface $\widetilde{V}$. Moreover, in terms of local coordinates

$$
w^{\prime}=w, \quad x^{\prime}=x / w \quad \text { and } \quad u^{\prime}=u / w
$$

on $\widetilde{V}$, the pullback of the form $\theta$ to $\widetilde{V}$ may be written as

$$
\begin{gathered}
\widetilde{\theta}=\frac{d\left(u^{\prime} w^{\prime}\right) \wedge d\left(x^{\prime} w^{\prime}\right)}{w^{\prime}} \\
=w^{\prime}\left(d u^{\prime} \wedge d x^{\prime}\right)+x^{\prime}\left(d u^{\prime} \wedge d w^{\prime}\right)+u^{\prime}\left(d w^{\prime} \wedge d x^{\prime}\right)
\end{gathered}
$$

which is regular on all of $\tilde{V}$. Thus all the forms $\theta_{k, \ell}$ above pull back to regular forms on $\widetilde{V}$. (It will also follow from more general results described in the next section that every regular differential on the smooth locus of $V$ gives a regular differential on $\widetilde{V}$.) $\widetilde{V}$ is thus a surface of general type, and so the family $X \rightarrow \mathbb{A}^{1}$ has correlation.

2.3. Adjoint conditions and canonical singularities. The example above contains the essential ingredients of the isotrivial case in general. There is one component in the analysis, however, that we will need to develop further in order to tackle arbitrary isotrivial families, and that is the way we handled the singularities of the surface $V$.

Let $X$ be an integral variety over a field $K$ of characteristic 0 . The condition that $X$ be of general type is defined in terms of pluricanonical forms on some desingularization

$$
\pi: \widetilde{X} \rightarrow X
$$

of $X$. But such resolutions may be hard to write down explicitly. There are times however, when the singularities of $X$ impose no impediment to the extension of pluricanonical sections from the smooth locus to any desingularization of $X$. Let us formalize this occurrence by the following definitions:

Definition 1. Let $X$ be any variety and $\pi: \widetilde{X} \rightarrow X$ a desingularization of $X$. By a smooth pluricanonical form on $X$ we will mean a pluricanonical form on the smooth locus of $X$ - that is, a section $\eta$ of the $m^{\text {th }}$ power of the canonical bundle of $X_{\mathrm{sm}}$ - such that the pullback $\pi^{*}(\eta)$ of $\eta$ to $\pi^{-1}\left(X_{\mathrm{sm}}\right) \subset \widetilde{X}$ extends to a regular pluricanonical form on all of $\widetilde{X}$. A point $p \in X$ is said to impose no adjoint conditions if for all $m$ and all sufficiently small neighborhoods $U$ of $p$ in $X$, any $m$-canonical form $\eta$ on the smooth locus $U_{\text {sm }}$ of $U$ is smooth.

Remark. Both of these notions are independent of the choice of resolution $\pi: \widetilde{X} \rightarrow$ $X$. Also, we should mention that the classical use of the phrase "imposes no adjoint conditions" was in the context of hypersurface singularities, where the condition above is satisfied for one value of $m$ if and only if it is satisfied for all $m$. It was, correspondingly, expressed in terms of canonical, rather than pluricanonical, differentials. 
We wish to relate this notion of imposing no adjoint conditions to the currently more common notion of canonical singularities. For this, recall that a point $p$ on a normal variety $X$ is $\mathbb{Q}$-Gorenstein if, in some neighborhood $U$ of $p$, some positive power $\left(K_{U_{\mathrm{sm}}}\right) \otimes m$ of the canonical bundle on the smooth locus of $U$ extends to a line bundle $L$ on all of $U$ (note that, since the singular locus of $X$ is of codimension at least $2, L$ is unique if it exists). In this case, we can make a direct comparison between the pullback of $L$ to $\widetilde{U}=\pi^{-1}(U) \subset \widetilde{X}$ and the corresponding pluricanonical bundle $\left(K_{U_{\mathrm{sm}}}\right)^{\otimes m}$ of $\widetilde{U}$ : we write

$$
\left(K_{\widetilde{U}}\right)^{\otimes m}=\pi^{*} L \otimes \mathcal{O}_{\widetilde{U}}(D)
$$

where $D$ is a divisor supported on the exceptional locus of the map $\pi$.

Definition 2. A $\mathbb{Q}$-Gorenstein point $p \in X$ is said to be a $t$ canonical singularity if the divisor $D$ occurring in (1) is effective.

We claim that in case $X$ is $\mathbb{Q}$-Gorenstein (i.e. some power of $K_{X_{\mathrm{sm}}}$ extends to a line bundle on the whole of $X$ ) this is equivalent to the condition that $p$ impose no adjoint conditions. To see this, in one direction let $\theta$ be any regular section of $\left(K_{U_{\mathrm{sm}}}\right)^{\otimes m}$. Since the singularities of $X$ occur in codimension at least $2, \theta$ will extend to a regular section of $L$. The pullback of this section will then be a rational section $\widetilde{\theta}$ of $\left(K_{\widetilde{U}}\right)^{\otimes m}$, whose divisor will be simply the pullback of the divisor of $\theta$, plus $D$. Thus, if $D$ is effective, then $(\widetilde{\theta})$ will be effective, i.e., $\widetilde{\theta}$ will be regular. Conversely, if $D$ is not effective a section of $L$ non-zero in a neighborhood of $p$ will give a rational differential on $\widetilde{U}$ with actual poles, whose restriction to $U_{\mathrm{sm}}$ is regular.

We should say at this point that we do not need to deal with these notions in anything like their full generality. In fact, all the singularities that we will be considering are quotients of local complete intersection varieties by finite groups. In particular, in this case the singularities will always be $\mathbb{Q}$-Gorenstein, so the reader can equate the notions of "imposing no adjoint conditions" and "canonical singularities". It is also the case that we have a very effective and elementary criterion for such singularities to be canonical, which we will prove in Section 4 (cf. Lemma 4.1). A Corollary of this Lemma that we will use here is

Corollary 2.1. Let $G$ be a finite group acting on a smooth variety $Y$, and $p \in Y$ an isolated fixed point of the action of $G$ - that is, an isolated fixed point of the action of every $\gamma \neq I d \in G$. If the dimension of $Y$ is greater than or equal to the order of $G$, then the image of $p$ in the quotient $X=Y / G$ imposes no adjoint conditions.

We should mention at the same time that this Corollary also follows from the more general Reid-Tai criterion, which we shall state here without proof. (See $[R]$.)

The Reid-Tai Criterion. Let $G$ be a finite group acting on a smooth variety $Y$, and $p \in Y$ a fixed point of the action of $G$. Suppose that the action satisfies the following condition: for all $\gamma \in G$ and primitive $m^{\text {th }}$ roots of unity $\zeta$, if the eigenvalues of the action $d \gamma$ of $\gamma$ on the tangent space $T_{p} Y$ are $\zeta^{a_{1}}, \cdots, \zeta^{a_{n}}$ with $0 \leq a_{i}<m$, then the exponents $a_{i}$ satisfy

$$
\sum a_{i} \geq m
$$

Then the singularity of the quotient $Y / G$ at the image $q$ of $p$ is canonical. 
2.4. Proof of Correlation for isotrivial families. Let $C$ be a smooth curve of genus $g \geq 2$, with automorphism group $G$, and suppose $X \rightarrow B$ is any isotrivial family with general fiber isomorphic to $C$. Of course, if the family is birationally a product, $X$ maps to $C$ and we are done. In general, however, we have to make a base change before the family becomes birationally trivial: explicitly, we may set

$$
B_{0}=\left\{(b, \psi): b \in B, \psi: X_{b} \rightarrow C \text { an isomorphism }\right\}
$$

and let $B_{1}$ be any connected component of $B_{0}$, dominating $B$. The pullback

$$
\begin{aligned}
X_{1} & =X \times_{B} B_{1} \\
& =\left\{(b, \psi, p):(b, \psi) \in B_{1}, p \in X_{b}\right\}
\end{aligned}
$$

is then visibly isomorphic to the product $B_{1} \times C$, via the maps $\pi_{1}:(b, \psi, p) \longmapsto$ $(b, \psi)$ and $\pi_{2}:(b, \psi, p) \longmapsto \psi(p)$. Moreover, the group $G$ acts on $B_{0}$ by setting for every $\gamma \in G$

$$
\gamma:(b, \psi) \longmapsto(b, \gamma \circ \psi) .
$$

The quotient $B_{0}$ under this action is $B$; let $H \subseteq G$ be the subgroup carrying $B_{1}$ into itself. $H$ also acts on $X_{1}$ by the rule

$$
\gamma:(b, \psi, p) \longmapsto(b, \gamma \circ \psi, p)
$$

in terms of the identification of $X_{1}$ with $B_{1} \times C$ above this is just the diagonal action of $H$ on $B_{1} \times C$

$$
\gamma:(b, \psi, q) \longmapsto(b, \gamma \circ \psi, \gamma(q))
$$

coming from the separate actions of $H$ on $B_{1}$ and on $C$.

The quotient of $X_{1}$ by $H$ is, by the first representation, birational to $X$ itself; from the second description we see that the quotient $X_{1} / H$ admits a map to the quotient $C / H$. Thus $X$ dominates the quotient $C / H$, and if this is a curve of genus 2 or more we are done. This is not, however, generally the case (as it is not in the example we started with).

What ultimately saves us is that, just as $X$ admits a dominant rational map to $C / H$, so by the same construction the fiber powers $X_{B}^{n}$ of $X$ admit dominant rational maps to the quotients $C^{n} / H$, where $H$ acts diagonally on the product $C^{n}$. We will thus be done if we establish the

Lemma 2.1. Let $C$ be a smooth curve of genus $g \geq 2$, and $H \subseteq$ Aut $(C)$ any group of automorphisms of $C$. Then for $n \geq|H|$, the quotient $C^{n} / H$ is of general type.

Proof. To begin with, $C^{n}$ is certainly of general type; in fact, its canonical bundle is ample, and its canonical ring

$$
R=\bigoplus_{m} H^{0}\left(C^{n},\left(K_{C^{n}}\right)^{\otimes m}\right)
$$

is finitely generated of dimension $n+1$. It follows that the subring $R^{H}$ of pluricanonical forms on $C^{n}$ invariant under the action of $H$ is likewise of dimension $n+1$. The problem is that pluricanonical forms on $C^{n}$ invariant under $H$ do not necessarily descend to give pluricanonical forms on $C^{n} / H$ : in the case $n=1$, for example, invariant pluricanonical forms descend to give rational pluricanonical forms that may have poles at the branch points of the map $C \rightarrow C / H$; that is, at the images of fixed points of the action of $H$.

For large $n$, however, this will not cause any trouble. To begin with, we observe that the fixed points of elements $\gamma \in H$ on $C^{n}$ are just points of the form $\mathbf{p}=$ 
$\left(p_{1}, \cdots, p_{n}\right)$ where the $p_{i}$ are (not necessarily distinct) fixed points of $\gamma$. The point is, these are isolated fixed points; applying Corollary 2.1, we deduce that whenever $n \geq|H|$ the singularities of $C^{n} / H$ are canonical, and we are done.

We may now deduce, as a corollary, the main result of this section:

Proposition 2.1. Let $X \rightarrow B$ be any isotrivial family of curves whose general fiber is a smooth curve $C$ of genus $g \geq 2$. Then the family $X \rightarrow B$ has correlation. More specifically, $X_{B}^{n}$ admits a dominant rational morphism to the variety $C^{n} / A u t(C)$, which is of general type for $n \gg 0$.

\section{FAMilies With MAXIMAL VARIATION OF MODUli}

in this section we prove Correlation for complete families of stable curves having maximal variation of moduli. In fact we will show the stronger fact that high fiber products of such families are of general type.

3.1. Preliminaries. We start by recalling some basic facts about stable curves and their moduli spaces. Let $g \geq 2$. As usual, we let $M_{g}$ be the moduli space of smooth curves of genus $g$, which is a reduced and irreducible quasiprojective variety. $M_{g}$ is not complete, and we need to consider its Deligne-Mumford compactification, which we denote by $\bar{M}_{g}$. The projective variety $\bar{M}_{g}$ is reduced and irreducible and it contains $M_{g}$ as a dense subset. It is characterized as the moduli space of the so-called Deligne-Mumford stable curves of arithmetic genus $g$. We recall the definition: A Deligne-Mumford stable curve (briefly: stable curve) is a reduced connected curve having only ordinary double points as singularities (that is, nodes) and having only finitely many automorphisms. The restriction on the number of automorphisms is just saying that every smooth rational component of a stable curve intersects the remaining components in at least three points. It is important to remark that $\bar{M}_{g}$ and $M_{g}$ are only coarse moduli spaces; that is, there does not exist a universal family of curves over such spaces.

Given a family $f: X \longrightarrow B$ of stable curves, we obtain a canonical morphism

$$
\begin{aligned}
\phi: B & \longrightarrow \bar{M}_{g} \\
b & \mapsto\left[X_{b}\right],
\end{aligned}
$$

which sends a point $b \in B$ to the point of $\bar{M}_{g}$ classifying the stable curve $X_{b}$. We will refer to $\phi$ as the canonical morphism to moduli. We will say that a family as above has maximal variation of moduli (or simply maximal variation) if its canonical morphism is generically finite; that is, if there are no isotrivial subfamilies including a general fiber.

Next, in proving Correlation we need to know how to use our information about line bundles on the initial family to obtain results about line bundles on the fiber product. The lemma that we are now going to prove is a very general one, and it will provide us with the key information.

Let $f: X \rightarrow B$ be a morphism with integral general fiber, and denote by $X_{B}^{n}$ the irreducible component of the $n$-fold fiber product of $X$ over $B$, dominating $B$ (just as in 1.1.3). If $L$ is a line bundle on $X$, we denote by $L_{n}$ the line bundle on $X_{B}^{n}$ defined as

$$
L_{n}:=p_{1}^{*} L \otimes \cdots \otimes p_{n}^{*} L
$$

where $p_{i}: X_{B}^{n} \rightarrow X$ is the projection onto the $i^{\text {th }}$ factor. Let $f_{n}$ be the natural morphism from $X_{B}^{n}$ to $B$. 
Lemma 3.1. Let $X \rightarrow B$ be a flat, projective family of varieties. Let $L$ be an ample line bundle on $X$ and let $M$ be an arbitrary line bundle on $B$. Then there exists a positive integer $n_{0}$ such that for all $n \geq n_{0}$ the line bundle $L_{n} \otimes f_{n}^{*} M$ is ample on $X_{B}^{n}$.

Moreover, the same holds if we replace "ample" by "big".

Proof. Let us assume that $L$ is ample. Then there exists a positive integer $e$ such that $L^{\otimes e} \otimes f^{*} M$ is itself ample. Hence, for every curve $C$ in $X$, we have

$$
\operatorname{deg}_{C}\left(L^{\otimes e} \otimes f^{*} M\right) \geq 0 .
$$

We will now take $n_{0}=e+1$ and $n$ will be any integer greater or equal to $n_{0}$, as in our statement. We want to apply Kleiman's ampleness criterion with $L_{n}$ as ample bundle $A$ ( $L_{n}$ is ample because $L$ is). In other words, we want to show that there exists a positive constant $\epsilon$ such that for every curve $C$ in $X_{B}^{n}$, we have

$$
\operatorname{deg}_{C}\left(L_{n} \otimes f_{n}^{*} M\right) \geq \epsilon \operatorname{deg}_{C}\left(L_{n}\right) .
$$

This is not hard. Denote $C_{i}:=p_{i}(C)$ and let $d_{i}$ be the degree of $C$ over $C_{i}$. Then we can estimate

$$
\begin{aligned}
\operatorname{deg}_{C}\left(L_{n} \otimes f_{n}^{*} M\right) & =\frac{e}{n} \operatorname{deg}_{C} L_{n}+\frac{n-e}{n} \operatorname{deg}_{C} L_{n}+\operatorname{deg}_{C} f_{n}^{*} M \\
& =\frac{e}{n} \sum_{i=1}^{n} d_{i} \operatorname{deg}_{C_{i}} L+\operatorname{deg}_{C} f_{n}^{*} M+\frac{n-e}{n} \operatorname{deg}_{C} L_{n} \\
& \geq-\frac{1}{n} \sum_{i=1}^{n} d_{i} \operatorname{deg}_{C_{i}} f^{*} M+\operatorname{deg}_{C} f_{n}^{*} M+\frac{n-e}{n} \operatorname{deg}_{C} L_{n} \\
& =\frac{n-e}{n} \operatorname{deg}_{C} L_{n} .
\end{aligned}
$$

Our positive constant $\epsilon$ is set equal to $(n-e) / n$ and we deduce that $L_{n} \otimes f_{n}^{*} M$ is ample.

Now suppose $L$ is big. Since $X$ is projective, a line bundle $L$ on $X$ is big if and only if there exist an ample line bundle $A$ and an effective Cartier divisor $D$ on $X$, such that

$$
L=A \otimes \mathcal{O}_{X}(D) .
$$

Therefore the proof of the result for the big line bundle $L$ follows immediately from the same result being true for the ample line bundle $A$.

3.2. Proof of Correlation for families with maximal variation. We are now ready to prove our main Theorem for complete families of stable curves with maximal variation of moduli. Therefore, throughout this section, we will assume that $f: X \longrightarrow B$ is a proper morphism of integral varieties, whose general fiber is a smooth curve of genus at least two. Moreover, we will require that $B$ is projective.

We will prove Correlation for our family as an immediate consequence of the following stronger result:

Proposition 3.1. Let $f: X \longrightarrow B$ be a family of stable curves, over a projective base $B$. Assume that the family has maximal variation of moduli. Then there exists a positive integer $n$ such that the $n^{\text {th }}$ fiber product of $X$ over $B$ is of general type. 
Proof. To understand what goes into the proof, suppose for simplicity that $X$ and $B$ are smooth and let $K_{X}$ and $K_{B}$ be the canonical bundles of $X$ and $B$; then, if $\omega_{f}$ is the relative dualizing sheaf of $X$ over $B$, we have of course

$$
K_{X}=\omega_{f} \otimes f^{*} K_{B}
$$

Modulo some known facts about families of stable curves, it is not hard to see that $\omega_{f}$ has good positivity properties, hence what really prevents $X$ itself from being of general type is evidently the canonical bundle of $B$, which is completely arbitrary. Now the point is that taking higher and higher powers of $X$ over $B$ will allow the positivity of $\omega_{f}$ to overcome the negativity of $K_{B}$ (cf. Lemma 3.1).

With that said, the argument reduces simply to two statements, for each of which we refer the reader to the literature (for a self-contained account of both, see Section 3 of [CHM1]). The first is simply the positivity of the relative dualizing sheaf:

First statement. Let $f: X \longrightarrow B$ be a family of stable curves having maximal variation of moduli. Then the relative dualizing sheaf $\omega_{f}$ is big.

The key ingredient in a proof of this is the fact that if $f: X \longrightarrow B$ is a family of stable curves having maximal variation of moduli, then the divisor class $\lambda$ - that is, the determinant of the direct image of the relative dualizing sheaf $\omega_{f}$ - is big. This is expressed in Proposition 7.1 of [V1].

Given this, we can write $\lambda=\alpha+D$ for some ample divisor class $\alpha$ and effective divisor class $D$ on $B$. Moreover, by a standard calculation due to Arakelov [Ar] and quoted in [V1], the divisor class $\omega_{f}$ on $X$ may be expressed as a sum of the pullback $f^{*} \lambda$ and an effective divisor: specifically, we have

$$
\frac{g(g+1)}{2} \omega_{f}=f^{*} \lambda+W
$$

where $W$ is the class of the divisor of Weierstrass points of fibers of $f$, plus a linear combination of the divisors of singular fibers of $f$.

Now, since $\omega_{f}$ is ample on every fiber of $f$, for sufficiently large $m$ the divisor class

$$
\beta=m \cdot f^{*} \alpha+\omega_{f}
$$

is ample on $X$. Combining this with the Arakelov relation (multiplied by $m$ ) we have

$$
\left(m \frac{g(g+1)}{2}+1\right) \omega_{f}=m \cdot f^{*} \alpha+\omega_{f}+m \cdot f^{*} D+m \cdot W=\beta+E .
$$

Thus $\omega_{f}$ is big, being the sum of an ample class and an effective class.

Notice now that the dualizing sheaf of the space $X_{B}^{n}$ may be expressed as a tensor product

$$
\omega_{X_{B}^{n}}=p_{1}^{*} \omega_{f} \otimes \ldots \otimes p_{n}^{*} \omega_{f} \otimes f_{n}^{*} K_{B} .
$$

Thus, the above statement together with our technical Lemma 3.1 implies that the dualizing sheaf of some high fiber power $X_{B}^{n}$ is big; that is, it has lots of sections. It remains to see that these give rise to sections of the canonical bundle of a desingularization of $X_{B}^{n}$, which constitutes the second part of the proof.

Second statement. Let $f: X \rightarrow B$ be any family of stable curves such that $B$ is smooth, irreducible and the support $\Delta \subset B$ of the discriminant locus of $f$ is a divisor with normal crossings. Then for every $n$, the singularities of the $n^{\text {th }}$ fiber power of $X$ over $B$ are canonical. 
This result can be easily proved by performing an explicit series of blow-ups and calculating the dualizing sheaf at each step. Alternatively, the referee of this paper points out that it may be proved using the arguments in [El]. The techniques of that paper show the following. Let $g: Y \rightarrow Z$ be a flat morphism such that $Z$ has rational singularities. Assume moreover that for any smooth curve $C$ and morphism $h: C \rightarrow Z$ whose image is not contained in the discriminant locus of $g$, the pullback $Y \times{ }_{Z} C$ has rational singularities. Then $Y$ has rational singularities.

Applying this inductively to the projection maps $X_{B}^{n} \rightarrow X_{B}^{n-1}$ we arrive at the desired statement.

We can ensure that the hypotheses of the second statement are satisfied by pulling our family back to a suitable blow-up of the original base $B$; this does not alter the fact that the family has maximal variation.

Now, since the singularities of $X_{B}^{n}$ are canonical, every regular section of $\omega_{X_{B}^{n}}$ pulls back to a regular section of a desingularization $\widetilde{X}$ of $X_{B}^{n}$. It follows that $K_{\tilde{X}}$ is big, which completes the proof of Proposition 3.1.

In fact the proof of Proposition 3.1 tells us something more, which will be necessary in the following sections; we will state it here as a lemma. To begin with, denote by $\widetilde{X_{B}^{n}}$ a desingularization of $X_{B}^{n}$ and by $\sigma$ the corresponding birational morphism from $\widetilde{X_{B}^{n}}$ to $X_{B}^{n}$. Also, let $g_{n}=f_{n} \circ \sigma$ denote the composition map

$$
g_{n}: \widetilde{X_{B}^{n}} \longrightarrow B \text {. }
$$

We then obtain the following

Lemma 3.2. Let $X \rightarrow B$ be a family of stable curves having maximal variation of moduli, and let $M$ be any line bundle on $B$. Then, with the above notation, there exists $n>0$ such that the line bundle

$$
\omega_{g_{n}} \otimes g_{n}^{*} M
$$

is big on $\widetilde{X_{B}^{n}}$.

\section{Families of Stable Curves With group action}

4.1. Statement of the main Proposition. In the preceding two sections we have considered families of curves representing two extremes of behavior: families of stable curves whose associated map to moduli is generically finite, and other families of curves for which it is constant. The techniques we used to analyze these cases were correspondingly different. In the former case we had our basic Proposition 3.1 on the positivity of the relative dualizing sheaf, combined with the argument that in sufficiently high fiber powers this positivity outweighs the possible negativity of the canonical bundle of the base (Lemma 3.1). In the isotrivial case, by contrast, we made a base change to trivialize the family, so that its fiber powers would map to the ordinary products of a curve with itself; we then had to analyze the geometry of the quotient of these products by the automorphism group of the curve.

We now want to consider the case of an arbitrary family. Since such a family in general involves curves of varying moduli, we will need to incorporate the content of Proposition 3.1 on the positivity of the relative dualizing sheaf. But such a Lemma deals only with families of stable curves, and in order to relate an arbitrary family 
to a family of stable curves, we will have to make a base change. Such a base change will have the effect of introducing a (finite) group action, of which we will have to consider the quotient. Moreover, since an arbitrary family will in general contain subfamilies that are isotrivial but not trivial, we will have to make base changes analogous to the one made in the isotrivial case to "straighten out" our family - and, if the original family is fibered by isotrivial subfamilies, express it as the pullback of a family over a lower-dimensional base. Again this means, exactly as in the isotrivial case, that at the end we will have to take a quotient of a variety of general type by a finite group and show that this does not lower its Kodaira dimension. We thus need a statement combining aspects of the isotrivial and the generically finite/stable cases; and this is what we will do here. Specifically, our goal in this section is to prove the

Proposition 4.1. Let $f: X \rightarrow B$ be a family of stable curves of genus $g \geq 2$ with projective base $B$ and smooth general fiber. Assume that the associated map $\phi: B \rightarrow \bar{M}_{g}$ is generically finite. Suppose $G$ is a finite group acting birationally and equivariantly on $X$ and $B$ - so that, for every $g \in G$ we have birational maps

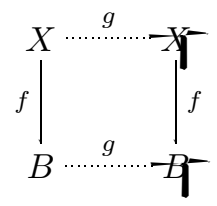

commuting with the projection. Then for all sufficiently large $n$, the quotient $X_{B}^{n} / G$ of $X_{B}^{n}$ by the action of $G$ acting diagonally is of general type.

In outline, the proof for this Proposition is simply a combination of the arguments already given, with one additional element. Briefly, Proposition 3.1 - which remains the mainspring of the proof - says that for $n \gg 0$ we have lots of sections of powers of the canonical bundle of $X_{B}^{n}$; and, as in the isotrivial case, it follows that we have lots of such sections invariant under $G$. The difference is that the fixed points of the action of $G$ on $X$ and on $X_{B}^{n}$ need not be isolated: in fact, since elements of $G$ may fix components of fibers of $f$, the codimension of the fixed point locus will not necessarily increase with $n$. Thus it will not always be the case, even for large $n$, that invariant differentials on $X_{B}^{n}$ descend to give smooth differentials on the quotient $X_{B}^{n} / G$; some vanishing at fixed points of the action of $G$ is required. This requirement, at least potentially, may kill off too many of our differentials.

What we need is a suitable bound on the order of vanishing needed to ensure that an invariant differential on $X_{B}^{n}$ descend to give a smooth differential on the quotient. This is the content of the following subsection, which also yields a proof of the weak version of the Reid-Tai criterion used in the isotrivial case (that is, Corollary 2.1).

4.2. Singularities of quotients: adjoint conditions. Let $X$ be a smooth, $n$ dimensional variety and $G$ a group of (finite) order $\ell$ acting on $X$; let $Y_{0}=X / G$ be the quotient. Let $\theta$ be an $m$-canonical form on $X$ invariant under the action of the group $G$, so that $\theta$ descends to give a pluricanonical form $\eta_{0}$ on the smooth locus of $Y_{0}$. We ask when the form $\eta$ is smooth. (Recall from Section 2 that by a smooth form on a possibly singular variety $Z$ we will mean a form on the smooth locus $Z_{\mathrm{sm}}$ of $Z$ whose pullback extends to a regular form on some, and hence any, 
desingularization.) A sufficient condition is given in

Lemma 4.1. For each point $p \in X$, let $G_{p} \subset G$ be the stabilizer of $p$ in $G$. If, for all points $p \in X, \theta$ vanishes to order at least $m \cdot\left(\left|G_{p}\right|-1\right)$ at $p$, then $\theta$ descends to a smooth form $\eta_{0}$ on $X / G$. In particular, if $\theta$ vanishes to order $m(\ell-1)$ on the locus of all points $p \in X$ that are fixed by some element $g \neq I d \in G$, then $\theta$ descends to a smooth form on $X / G$.

Proof. We start with a few observations.

First, the action of $G$ on $X$ is equivalent, in a neighborhood of a fixed point $p \in X$ (in the analytic or étale topology), to its action on the tangent space to $X$ at $p$; so we can simply replace $X$ by the vector space $V=T_{p}(X)$ and take the action to be a linear representation of $G$.

Second, we may decompose $V$ into a direct sum of irreducible representations of $G$ and throw away the trivial summands: if $V$ is the direct sum of subspaces $V^{\prime} \subset V$ and $V^{\prime \prime} \subset V$ with $G$ acting trivially on $V^{\prime \prime}$, it is enough to verify the statement for the action of $G$ on $V^{\prime}$. Thus we may assume that the origin $0 \in V$ is the only point of $V$ fixed by the action of all of $G$.

Third, for any fixed action of $G$ on $V$, there is some order of vanishing that works; that is, some number $\alpha(m)$ such that an $m$-canonical form $\theta$-invariant under $G$ and vanishing to order $\alpha(m)$ or more on the locus of fixed points of elements of $G$ descends to a smooth form on the quotient. (To see this, observe that if $Y$ is any desingularization of the quotient $V / G$, then pulling back to $V$ a set of generators for the direct image in $V / G$ of the pluricanonical bundle of $Y$ we obtain a collection of pluricanonical forms on $V$ that generate a subsheaf of the canonical bundle $K_{V}$. This subsheaf coincides with $K_{V}$ outside the locus $\Gamma$ of fixed points of elements of $G$ and so must contain the product of $K_{V}$ with some power of the ideal sheaf of $\Gamma$.) Since there are only finitely many groups of order $\ell$ or less and only finitely many representations of each such group on vector spaces of given dimension, there is a number that works for all actions of all groups of order $\ell$ or less on vector spaces of dimension $n$ or less. We will denote by $\beta=\beta(m, n, \ell)$ the minimal such number.

Our goal is thus to show that $\beta \leq m(\ell-1)$. Note that we cannot have $\beta<$ $m(\ell-1)$, as shown by the simple example of a cyclic group of order $\ell$ acting on a one-dimensional vector space $V$. In fact, we will see in the proof that this is the unique case where a form has to vanish to order $m(\ell-1)$ in order to descend to a smooth form; for any other action of a group of order $\ell$ on a vector space (without any trivial factors) a lower order of vanishing suffices.

Let $\theta$ be any $m$-canonical form on $V$ invariant under $G$ and satisfying the hypothesis of the Lemma; let $\eta_{0}$ be the $m$-canonical form induced on the smooth locus of the quotient $Y_{0}=V / G$ and $\eta$ its extension to a rational $m$-canonical form on a resolution $\pi: Y \rightarrow Y_{0}$. By our hypothesis that the action of $G$ on $V$ contains no trivial factors, the stabilizer of any point $p \in V$ other than the origin is a proper subgroup of $G$. By induction on the order $\ell$, then, we may assume that $\eta$ is regular outside the inverse image $\pi^{-1}(\overline{0}) \subset Y$ of the image $\overline{0}$ of the origin $0 \in V$, and we just have to check the regularity of $\eta$ at points of $\pi^{-1}(\overline{0})$.

There are now two cases to consider: either $n=1$, or it isn't.

If $n=1$, things are simple: the group acts on the one-dimensional vector space $V$ by scalar multiplication by $\ell^{\text {th }}$ roots of unity; the quotient $Y=Y_{0}=V / G$ is again smooth, with the quotient map $V \rightarrow Y$ a cyclic cover of order $\ell^{\prime} \leq \ell$ ramified at the origin $0 \in V$. A regular $m$-canonical differential on $Y$ pulls back to one on 
$V$ with a zero of order $m \cdot\left(\ell^{\prime}-1\right)$, and conversely an invariant differential on $V$ descends to a regular form on $Y$ if it vanishes to order $m \cdot\left(\ell^{\prime}-1\right)$ or more at 0 .

If $n>1$, on the other hand, let $\widetilde{V}$ be the blow up of the origin in $V$; the action of $G$ on $V$ lifts to an action of $G$ on $\widetilde{V}$, and we have a diagram

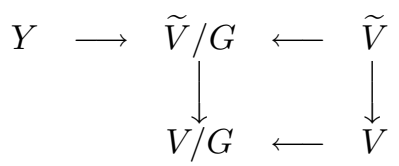

where $Y$ is any resolution of singularities of $\widetilde{V} / G$, and hence of $V / G$ as well.

Now let $\theta$ be any $m$-canonical form on $V$ invariant under $G$, and $\widetilde{\theta}$ the pullback to $\theta$ to $\widetilde{V}$; let $\eta$ and $\widetilde{\eta}$ be the forms induced on the smooth loci of $V / G$ and $\widetilde{V} / G$ respectively. Since $\eta$ pulls back to $\widetilde{\eta}$ on $\widetilde{V} / G$ and both pull back to the same form on the desingularization $Y$, we see that $\theta$ descends to a smooth form if and only if $\widetilde{\theta}$ does. On the other hand, if $\gamma$ is the order of vanishing of $\theta$ at the origin $0 \in V$, then the order of vanishing of $\widetilde{\theta}$ at every point of the exceptional divisor $E \subset \widetilde{V}$ is $\gamma+m(n-1)$.

Combining the two cases, we conclude that $\beta=\beta(m, n, \ell)$ satisfies

$$
\beta \leq \max \{m \cdot(\ell-1), \beta-m(n-1)\}
$$

and hence

$$
\beta=m \cdot(\ell-1)
$$

We should remark that Lemma 4.1 gives, as an immediate corollary, the weak form of the Reid-Tai Criterion (Corollary 2.1) used in Section 2. Another corollary is the following statement, which was suggested by Viehweg:

Corollary 4.1. Let $X$ be any variety of general type and $G$ a finite group of birational automorphisms of $X$. Then for $n \gg 0$, the quotient $X^{n} / G$ of $X^{n}$ by $G$ (acting diagonally) is of general type.

Proof. As in the proof of Proposition 4.1 below, we may assume both that $X$ is smooth and that the action of $G$ is biregular. We then simply observe that the codimension in $X^{n}$ of the locus of points fixed by $g \in G$ is at least $n$.

We will want to apply Lemma 4.1 to the total space of the fiber powers of a family of curves. These varieties may not be smooth, but their singularities will be canonical; therefore we will need the following slight extension of Lemma 4.1.

Corollary 4.2. Let $X$ be a variety with canonical singularities and $G$ a finite group of biregular automorphisms of $X$. Let $\theta$ be a G-invariant, regular section of the $m^{\text {th }}$ power of the dualizing sheaf of $X$, such that for every point $p \in X, \theta$ vanishes to the order at least $m \cdot\left(\left|G_{p}\right|-1\right)$ at $p$. Then $\theta$ descends to a regular $m$-canonical form on the quotient $X / G$.

Proof. We first need to introduce an equivariant desingularization of $X$; that is, a resolution $\sigma: \widetilde{X} \longrightarrow X$ such that the action of $G$ on $X$ lifts to an action on $\widetilde{X}$. The existence of such a resolution is asserted in the survey article [Hi], which formulates a number of important results regarding resolution of singularities. For a proof in the special context of hypersurfaces (which is, in fact, not our context) see [BM]. 
Now observe that the stabilizer of any point $q \in \widetilde{X}$ is a subgroup of the stabilizer of its image $\sigma(q)=p$. At the same time, since the singularities of $X$ are canonical, we have

$$
\omega_{\tilde{X}}=\left(\sigma^{*} \omega_{X}\right)(E)
$$

where $E$ is an effective divisor. We conclude that if $\theta$ is any $m$-canonical form on $X$, the order of vanishing of its pullback $\widetilde{\theta}$ to $\widetilde{X}$ at $q$ will be at least equal to the order of vanishing of $\theta$ at $q$. Thus if $\theta$ satisfies the hypotheses of our statement, then so will $\widetilde{\theta}$.

Now let us denote by $Y$ the quotient $X / G$ and let $\tilde{Y}=\tilde{X} / G$. Finally, let $Y^{\prime}$ be any resolution of singularities of $\widetilde{Y}$ :

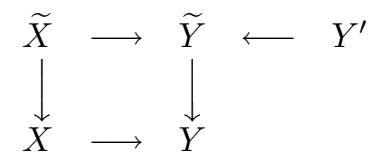

Suppose that $\theta$ is an $m$-canonical form on $X$, invariant under the action of $G$. The form $\theta$ descends to a form $\eta$ on the smooth locus of $Y$; let $\widetilde{\eta}$ be the pullback of $\eta$ to $\widetilde{Y}$. We can also view $\tilde{\eta}$ as the descent of $\widetilde{\theta}$ from $\widetilde{X}$. If $\theta$ satisfies the hypothesis of the Corollary, so does $\widetilde{\theta}$; since we have proved the statement for a smooth variety (Lemma 4.1), it follows that the pullback of $\widetilde{\eta}$ to $Y^{\prime}$ will extend to a global regular form.

4.3. Proof of the main Proposition and other corollaries. Here is the main application of Lemma 4.1.

Proof of Proposition 4.1. Let us review the context of the Proposition: we have a family $f: X \rightarrow B$ of stable curves, with projective base $B$ and smooth general fiber, whose associated map $\phi: B \rightarrow \bar{M}_{g}$ is generically finite, and on which a finite group $G$ acts birationally.

We claim that we may assume at the outset that our family $X \rightarrow B$ has smooth base $B$, that the discriminant locus $\Delta$ in $B$ (that is, the locus of points $b \in B$ such that the fiber $X_{b}$ is singular) has normal crossings, and that the action of $G$ on both $B$ and $X$ is regular. We achieve this state of affairs by making two base changes. First, we make a regular birational base change $B^{\prime} \rightarrow B$ to ensure that the action of $G$ on $B$ is biregular. To do this, we label the elements of $G$ as

$$
G=\left\{g_{1}, \cdots, g_{\ell}\right\}
$$

and take $U \subset B$ to be an open subset such that the birational map $g_{\alpha}: B \rightarrow B$ is regular on $U$ for all $\alpha$. We then take $B^{\prime}$ to be the closure of the locus

$$
\Gamma=\left\{\left(g_{1} p, \cdots, g_{\ell} p\right): p \in U\right\} \subset B^{\ell} ;
$$

and we observe that the action of $G$ on $B^{\prime}$ is regular, since it is just the restriction of the action of $G$ on $B^{\ell}$ permuting the factors.

Next, we make another base change $B^{\prime \prime} \rightarrow B^{\prime}$, with $B^{\prime \prime}$ a resolution of singularities of $B^{\prime}$, such that the support of the discriminant locus of the induced family $X^{\prime \prime}=X \times{ }_{B} B^{\prime \prime} \longrightarrow B^{\prime \prime}$ is a divisor with normal crossing. Moreover, by [Hi] we can do this equivariantly with respect to the action of $G$; that is, so that the action of $G$ on $B^{\prime}$ lifts to a regular action of $G$ on $B^{\prime \prime}$.

Finally, we claim that if $X^{\prime \prime}=X \times_{B} B^{\prime \prime}$ is the pullback of our family to $B^{\prime \prime}$, the action of $G$ on $X$ lifts to a regular action of $G$ on $X^{\prime \prime}$. This follows from the fact 
that stable curves have only finitely many automorphisms, which says that for any $g \in G$, the variety

$$
\Psi=\left\{(b, \psi): b \in B^{\prime \prime}, \quad \psi: X_{b} \rightarrow X_{g(b)} \quad \text { an isomorphism }\right\}
$$

is a finite cover of $B^{\prime \prime}$. The action of $g$ on $B^{\prime \prime}$ lifts over an open subset $U$ of $B^{\prime \prime}$, so that we have a section of $\Psi \rightarrow B^{\prime \prime}$ over $U$; since $\Psi \rightarrow B^{\prime \prime}$ is finite and $B^{\prime \prime}$ is smooth (and in particular normal), this section extends to all of $B^{\prime \prime}$.

We continue with the proof. We will proceed in two stages: we will give the proof first under the additional hypothesis that the action of $G$ on the base $B$ of our family is faithful; that is, no element $g \in G$ other than the identity fixes $B$ pointwise. This is, as we will see in the following section, the case relevant to families having maximal variation, and the proof is somewhat more transparent here. Finally, we will indicate the modifications necessary to make the argument work without this assumption.

Assuming that the action of $G$ on $B$ is indeed faithful, let

$$
\Phi=\{b \in B: g(b)=b \quad \text { for some } \quad g \neq I d \in G\}
$$

be the locus of points $b \in B$ fixed by some element $g \in G$ other than the identity, and let $D_{0}$ be any effective divisor on $B$ whose support contains $\Phi$. Set

$$
D=|G| \cdot D_{0}
$$

and let $M$ be the line bundle

$$
M=K_{B}(-D)
$$

By Lemma 3.2, for all sufficiently large $n$ the line bundle

$$
\omega_{f_{n}} \otimes f_{n}^{*} M
$$

is big; choose such an integer $n$. We see then that the ring

$$
R=\bigoplus_{m=0}^{\infty} H^{0}\left(X_{B}^{n},\left(\omega_{f_{n}} \otimes f_{n}^{*} M\right)^{m}\right)
$$

of sections of powers of $\omega_{f_{n}} \otimes f_{n}^{*} M$ has (maximal) dimension $\operatorname{dim}\left(X_{B}^{n}\right)+1$, and correspondingly so does the subring $R^{G}$ of elements of $R$ fixed under the action of $G$. But now we can write

$$
\omega_{f_{n}} \otimes f_{n}^{*} M=\omega_{X_{B}^{n}}\left(-|G| \cdot f_{n}^{*} D_{0}\right)
$$

so we can view sections of $\left(\omega_{f_{n}} \otimes f_{n}^{*} M\right)^{m}$ as $m$-canonical differentials on $X_{B}^{n}$ vanishing to order $m \cdot|G|$ along the locus of points of $X_{B}^{n}$ fixed by elements of $G$ other than the identity. Thus, applying Corollary 4.2 we see that if $Z$ is any resolution of singularities of $X_{B}^{n} / G$, we have an inclusion

$$
R^{G} \hookrightarrow \bigoplus_{m=0}^{\infty} H^{0}\left(Z,\left(K_{Z}\right)^{m}\right)
$$

of $R^{G}$ in the canonical ring of $Z$. We conclude finally that $Z$ is of general type, and hence so is the quotient $X_{B}^{n} / G$. 
It remains to consider what may happen if the subgroup $G^{\prime} \subset G$ of elements of $G$ that fix $B$ pointwise is not equal to the identity. Now, if an element $g \in G$ that acts trivially on $B$ does not fix pointwise any component of a fiber of $X$ over $B$, then the codimension of its fixed point locus in $X_{B}^{n}$ grows with $n$, and there is no problem. We thus have to replace $\Phi \subset B$ above with the subset

$$
\Phi=\left\{b \in B: g \text { fixes some component of } X_{b} \text { pointwise, for some } g \neq I d \in G\right\},
$$

and again let $D_{0}$ be any effective divisor on $B$ whose support contains $\Phi$; the argument then proceeds as before.

\section{The general CASE}

5.1. Stable reduction over arbitrary bases. We are now prepared to complete the proof of Theorem 1.3, which says that if $f: X \rightarrow B$ is an arbitrary family of curves (by which we mean any morphism of integral varieties whose general fiber is a smooth curve of genus $g \geq 2$ ), then high fiber powers of $X$ over $B$ admit dominant rational maps to varieties of general type. To do this, we have to relate our arbitrary family to the sort of families considered in Proposition 4.1 above, so that we can deduce our Theorem using Proposition 4.1.

Given an arbitrary family $f: X \rightarrow B$ of curves, we want to construct a family of stable curves whose smooth fibers are those of the family $f$. In other words, we want to carry out stable reduction over an arbitrary-dimensional base $B$. A key step in doing this is the following

Lemma 5.1 (Tautological families of stable curves). There exists a tautological family over a finite cover of the moduli space of stable curves; that is, there exists a variety $\Omega$ and a family of stable curves $\mathcal{T} \rightarrow \Omega$ such that the associated map $\varphi: \Omega \rightarrow \bar{M}_{g}$ is finite and surjective. Equivalently, there exists a variety $\Omega$, a finite surjective map $\varphi: \Omega \longrightarrow \bar{M}_{g}$ and a family of stable curves $\mathcal{T} \rightarrow \Omega$ such that $\varphi(x)=\left[T_{x}\right]$ for every $x$ in $\Omega$.

Remark. This follows from the existence of universal curves with suitably defined level structure; see [Po1] or [Po2]. It is also true for more general moduli problems; see [Ko1]. In the special case of curves Looijenga and Pikaart (see for example [Lo]) have given explicit constructions of such $\Omega$ showing in particular that we can take $\Omega$ to be smooth. The constructions of Looijenga and Pikaart, however, are subtle, and require a deep understanding of the local structure of families of stable curves. Alternatively, one can give an ad-hoc proof of just this statement, using the description of $\bar{M}_{g}$ as a geometric quotient (in the sense of geometric invariant theory) of the Hilbert scheme $H_{g}$ parametrizing $n$-canonically embedded stable curves (cf. Section 1.2). Since $H_{g}$ is naturally endowed with a universal family of curves, a tautological family can be constructed by patching together local liftings of the map $H_{g} \rightarrow \bar{M}_{g}$; for the details, see Section 5 of [CHM1].)

As we indicated, the import of Lemma 5.1 for us is the following Corollary. (The proof of this statement will be carried out in the course of the proof of the Correlation Theorem 1.3 in the next section; but it seems worth stating on its own.) Before we state it, however, we should make one remark concerning base change.

Remark. Suppose we are given a family $f: X \rightarrow B$ of curves, and we want to apply to this family the base change associated to a generically finite map $B^{\prime} \rightarrow B$ 
with $B^{\prime}$ irreducible. If in fact the fiber dimension of $f$ does jump, then it may happen that the fiber product $X \times_{B} B^{\prime}$ is no longer irreducible. In this case, we will simply disregard the components of the fiber product that fail to dominate $B$ : we define the essential pullback $X^{\prime} \rightarrow B^{\prime}$ of our family to be the unique irreducible component $X^{\prime}$ of the fiber product $X \times_{B} B^{\prime}$ dominating $B$, with the restriction of the projection map to $B^{\prime}$.

Corollary 5.1 (Stable reduction for curves). Given any morphism $f: X \rightarrow B$ of integral varieties whose general fiber is a smooth curve of genus $g \geq 2$, there exists a generically finite map $B^{\prime} \rightarrow B$, a family of stable curves $X^{\prime} \rightarrow B^{\prime}$ and a birational isomorphism of $X^{\prime}$ with the essential pullback of the family $X \rightarrow B$ to $B^{\prime}$.

5.2. Completion of the proof of the Correlation Theorem 1.3. We consider now the general case: an arbitrary morphism $f: X \rightarrow B$ of integral varieties whose general fiber is a smooth connected curve of genus $g \geq 2$. We will not assume anything about the smoothness or singularity of the spaces $X$ or $B$, or about the flatness (or even constancy of fiber dimension) of the map.

Our first goal must be, starting with the family $f: X \rightarrow B$, to find a family $\mathcal{T}_{f} \rightarrow$ $\Sigma$ of stable curves that $X$ dominates, and to which we can apply Proposition 4.1 of the preceding section. We cannot do this right away, however; we first have to make a series of base changes of the original family to obtain families $X_{i} \rightarrow B_{i}$, and then at the end we need to deduce the desired statement (that for $n$ sufficiently large the fiber power $X_{B}^{n}$ dominates a variety of general type) for the original family, not the one obtained after base change. Note that since the statement we wish to make (that $X_{B}^{n}$ dominates a variety of general type for $n \gg 0$ ) concerns only the birational isomorphism class of our original family $X \rightarrow B$, we do not need to worry about particular models for the families $X_{i} \rightarrow B_{i}$; for example, we may specify a base change as a dominant rational map $B_{i+1} \rightarrow B_{i}$ - that is, only on an open subset of $B_{i}$. We cannot allow ourselves the same luxury with regard to the family $\mathcal{T}_{f} \rightarrow \Sigma$, however: if we want to apply Proposition 4.1 to it, we need to know that $\mathcal{T}_{f} \rightarrow \Sigma$ is a complete family of stable curves.

First base change. By hypothesis there is an open subset $U \subset B$ over which the map $f$ is smooth, with fibers smooth connected curves of genus $g$. We thus have a regular map $U \rightarrow \bar{M}_{g}$, which extends to a rational map $B \rightarrow \bar{M}_{g}$. We let $B_{1}$ be the graph of this map, and $\Sigma_{1}$ its image in $\bar{M}_{g}$; let $X_{1} \rightarrow B_{1}$ be the essential pullback of our family to $B_{1}$.

Note that the map $B_{1} \rightarrow \Sigma_{1} \subset \bar{M}_{g}$ determines, for every point $p \in B_{1}$, a stable curve, which is isomorphic to the fiber of $X_{1}$ over $b$ for an open subset of $b \in B_{1}$. But these stable curves do not necessarily fit together to form a family; for that we have to pass to a cover of $\Sigma_{1}$ and the corresponding cover of $B_{1}$.

Second base change. Now, we have constructed a tautological family over the cover $\varphi: \Omega \longrightarrow \bar{M}_{g}$ in Lemma 5.1. We then let $\Sigma_{2}=\varphi^{-1}\left(\Sigma_{1}\right)$ be the inverse image of $\Sigma_{1}$, and $\mathcal{T}_{2} \longrightarrow \Sigma_{2}$ the restriction of the tautological family $\mathcal{T} \longrightarrow \Omega$ to $\Sigma_{2} \subset \Omega$. We then make the corresponding base change on our family $X_{1} \longrightarrow B_{1}$; that is, we set

$$
B_{2}=B_{1} \times_{\Sigma_{1}} \Sigma_{2}
$$

and let $X_{2} \longrightarrow B_{2}$ be the essential pullback of our family to $B_{2}$. We arrive at the 
diagram

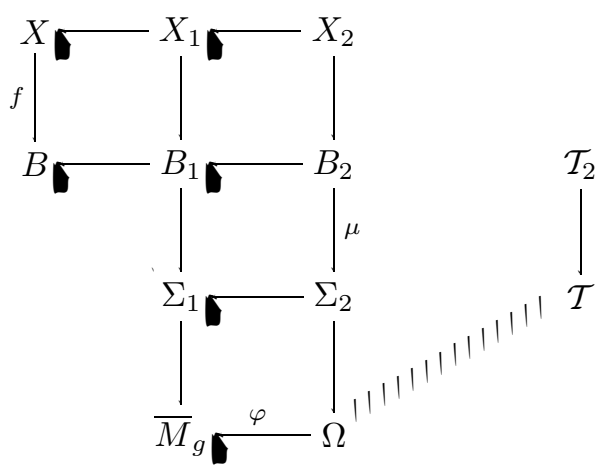

Third base change. We now have a family $\mathcal{T}_{2} \rightarrow \Sigma_{2}$ of stable curves, and a map $B_{2} \rightarrow \Sigma_{2}$ such that, on an open subset $U$ of $B_{2}$ at least, the fiber of our pullback family $X_{2} \rightarrow B_{2}$ over each $b \in U \subset B_{2}$ agrees with (that is, is isomorphic to) the fiber of $\mathcal{T}_{2}$ over the image of $b$ in $\Sigma_{2}$. This is not enough, however: we would like to say that the family $X_{2} \rightarrow B_{2}$ is birational to the pullback of the family $\mathcal{T}_{2} \rightarrow \Sigma_{2}$ to $B_{2}$; but the fact that the corresponding fibers are individually isomorphic is not sufficient to guarantee this. For example, consider the isotrivial case. If the original family $X \rightarrow B$ were isotrivial with general fiber $C$, the map $B \rightarrow \bar{M}_{g}$ would be constant, and all the constructions made so far essentially trivial: we would have $\Sigma_{2}=\Sigma_{1}=\{[C]\}, B_{2}=B_{1}=B$ and $X_{2}=X_{1}=X$; but the family $X \rightarrow B$ need not be, as we observed before, birationally trivial.

We thus have to make one more base change in order to arrive at a family $X_{3} \rightarrow B_{3}$ that is indeed birational to the pullback of a family $\mathcal{T}_{3} \rightarrow \Sigma_{3}$ of stable curves finite over moduli. We do this exactly as in the isotrivial case: for every point $b \in B_{2}$ we consider the set of isomorphisms between the fiber of $X_{2}$ over $b$ and the fiber of the pullback of $\mathcal{T}_{2} \rightarrow \Sigma_{2}$ to $b$, that is, the fiber of $\mathcal{T}_{2}$ at $\mu(b)$, where $\mu$ is the base change $\mu: B_{2} \longrightarrow \Sigma_{2}$; over an open subset of $B_{2}$ these will give us our cover $B_{3} \rightarrow B_{2}$. The one difference is that, since we want a finite group to act on $X_{3} \rightarrow B_{3}$ with quotient $X \rightarrow B$, we may have to make one further extension to ensure that the generically finite map $B_{3} \rightarrow B$ is Galois, i.e., that the extension of function fields $K\left(B_{3}\right) / K(B)$ is Galois.

Explicitly, we set

$$
B^{\prime}=\left\{(b, \psi): b \in B_{2}, \quad \psi:\left(X_{2}\right)_{b} \longrightarrow\left(\mathcal{T}_{2}\right)_{\mu(b)} \quad \text { an isomorphism }\right\}
$$

and let $B^{\prime \prime}$ be any connected component of $B^{\prime}$ dominating $B_{2}$; we let $B_{3}$ be the Galois normalization of $B^{\prime \prime} \rightarrow B$ (that is, the normalization of $B$ in the Galois closure of the function field of $\left.B^{\prime \prime}\right)$. If $U \subset B_{2}$ is an open subset where the fibers $\left(X_{2}\right)_{b}$ and $\left(\mathcal{T}_{2}\right)_{\mu(b)}$ are indeed isomorphic, and the automorphism group of $\left(X_{2}\right)_{b}$ is constant, then over $U$ the pullback

$$
\begin{aligned}
X_{3} & =X_{2} \times_{B_{2}} B_{3} \\
& =\left\{(b, \psi, p):(b, \psi) \in B_{3}, p \in X_{b}\right\}
\end{aligned}
$$

of our family to $B_{3}$ is visibly isomorphic to the pullback $B_{3} \times_{\Sigma_{2}} \mathcal{T}_{2}$, via the maps $\pi_{1}:(b, \psi, p) \longmapsto(b, \psi)$ and $\pi_{2}:(b, \psi, p) \longmapsto \psi(p)$.

By construction, we have an action of the Galois group

$$
G=\operatorname{Gal}\left(K\left(B_{3}\right) / K(B)\right)
$$


on $B_{3}$, with quotient birational to $B$. Since $X_{3}$ is birationally the pullback $X \times_{B} B_{3}$, that action lifts as well to a birational action of $G$ on $X_{3}$, with quotient $X_{3} / G$ birational to the total space $X$ of our original family.

Finally, we introduce our desired family $\mathcal{T}_{3} \rightarrow \Sigma_{3}$. To start with the base $\Sigma_{3}$, we want this to be in some sense the finite cover of $\Sigma_{2}$ analogous to the cover $B_{3} \rightarrow B_{2}$ - that is, we want the map $B_{3} \rightarrow \Sigma_{2}$ to factor through it, with the same finite group $G$ acting simultaneously on $B_{3}$ and $\Sigma_{3}$, with quotients birational to $B_{1}$ and $\Sigma_{1}$; we would also like that action to lift to the pullback $\mathcal{T}_{3}=\mathcal{T}_{2} \times_{B_{2}} B_{3}$ as well; that is, we want $G$ to act (birationally) on the whole diagram

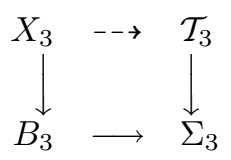

where the top arrow is the composition of the birational isomorphism $X_{2} \rightarrow B_{3} \times_{\Sigma_{2}}$ $\mathcal{T}_{2}$ with the projection $B_{3} \times_{\Sigma_{2}} \mathcal{T}_{2} \longrightarrow \Sigma_{3} \times_{\Sigma_{2}} \mathcal{T}_{2}=\mathcal{T}_{3}$.

There are several ways of characterizing the desired variety $\Sigma_{3}$. Algebraically, we can let $L$ be the algebraic closure of the function field $K\left(\Sigma_{2}\right)$ in the function field $K\left(B_{3}\right)$, and take $\Sigma_{3}$ to be the normalization of $\Sigma_{2}$ in the field $L$. Geometrically, we apply Stein factorization to the map $B_{3} \rightarrow \Sigma_{2}$ to express this as a composition $B_{3} \rightarrow \Sigma_{3} \rightarrow \Sigma_{2}$, where $\Sigma_{3} \rightarrow \Sigma_{2}$ is finite and $B_{3} \rightarrow \Sigma_{3}$ has connected fibers.

Either way, the main point to check is that the action of $G$ on $X_{3} \rightarrow B_{3}$ descends to a (birational) action of $G$ on $\mathcal{T}_{3} \rightarrow \Sigma_{3}$. From the algebraic description of $\Sigma_{3}$ it is clear that $G$ acts birationally on $\Sigma_{3}$ : since $G$ acts on the field $K\left(B_{3}\right)$ fixing the subfield $K\left(B_{1}\right)=K(B)$, it fixes $K\left(\Sigma_{1}\right) \subset K\left(B_{1}\right)$; thus it carries elements of $K\left(B_{3}\right)$ algebraic over $K\left(\Sigma_{1}\right)$ into elements algebraic over $K\left(\Sigma_{1}\right)$, which is to say it acts on the field $L=K\left(\Sigma_{3}\right)$. Geometrically, we can also see this readily: suppose we are given a general point $\sigma \in \Sigma_{3}$ and an element $g \in G$, and we want to decide to which of the (finitely many) points of $\Sigma_{3}$ in the fiber of $\Sigma_{3} \rightarrow \Sigma_{1}$ over $\sigma$ the action of $g$ should send $\sigma$. We simply choose a general point $b$ in the fiber of $B_{3} \rightarrow \Sigma_{3}$ over $\sigma$, and send $\sigma$ to the image of $g(b)$; since the general fiber of $B_{3}$ over $\Sigma_{3}$ is irreducible, there is no ambiguity in this choice. The same applies to the map $X_{3} \rightarrow \mathcal{T}_{3}$ : since the general fiber is irreducible, the action of $G$ on $X_{3}$ descends to an action on $\mathcal{T}_{3}$.

We arrive (finally!) at the diagram that contains the desired map $\nu$ :

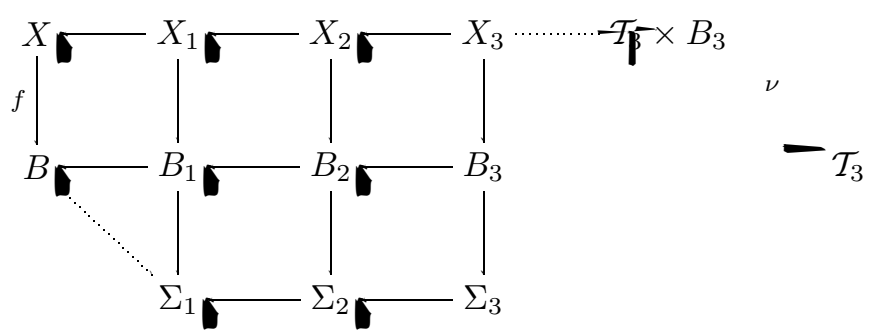

$$
\bar{M}_{g}
$$

The point is, the group $G$ acts as a group of birational automorphisms of $X_{3}$ and $\mathcal{T}_{3}$, and hence acts on the fiber powers $\left(X_{3}\right)_{B_{3}}^{n}$ and $\left(\mathcal{T}_{3}\right)_{\Sigma_{3}}^{n}$ as well. In the former case, the quotient $\left(X_{3}\right)_{B_{3}}^{n} / G$ is birational to the fiber power $X_{B}^{n}$ of our original 
family. We thus have a dominant map

$$
X_{B}^{n \stackrel{\cong}{-}}\left(X_{3}\right)_{B_{3}}^{n} / G \longrightarrow\left(\mathcal{T}_{3}\right)_{\Sigma_{3}}^{n} / G
$$

and by Proposition 4.1 of the preceding section, for $n$ sufficiently large the quotient $\left(\mathcal{T}_{3}\right)_{\Sigma_{3}}^{n} / G$ is of general type.

\section{The Higher-Dimensional CASE}

Having completed the proof of the Correlation Theorem, two questions naturally arise: is the analogous statement valid for families with higher-dimensional fibers, and what are the consequences if it is? We will discuss these questions in turn in this section.

6.1. Correlation for families of higher-dimensional varieties. For the first question, both János Kollár and Eckhart Viehweg have, in private correspondence with us, indicated that the likely answer is "yes"; we will state it as a conjecture and discuss which ingredients of a possible proof are known, and which represent potential obstacles. The discussion below is based to a large degree on our conversations with Kollár and Viehweg.

Conjecture H. Let $f: X \rightarrow B$ be an arbitrary morphism of integral varieties, whose general fiber is an integral variety of general type. Then for $n \gg 0, X_{B}^{n}$ admits a dominant rational map $h$ to a variety $W$ of general type such that the restriction of $h$ to a general fiber of $f$ is generically finite.

This has recently been proved by Brendan Hassett [Ha] in case the general fiber of $f$ is a surface.

To understand what is involved in a proof of Conjecture $\mathrm{H}$, it is useful to go back to the case of one-dimensional fibers, and even to the very first example we studied: the general pencil of plane quartics, which we described in Section 2.1. This time, however, take the family $X \rightarrow B$ of all plane quartics, parametrized by the projective space $B=\mathbb{P}^{14}$. The situation is formally very much the same: $X$ is a smooth hypersurface in $\mathbb{P}^{14} \times \mathbb{P}^{2}$, given as the zero locus of the (bihomogenization of the) polynomial

$$
\sum a_{i, j} x^{i} y^{j}=0
$$

which is to say, again a smooth hypersurface of bidegree $(1,4)$. Given that the canonical bundle of $\mathbb{P}^{14} \times \mathbb{P}^{2}$ is

$$
K_{\mathbb{P}^{14} \times \mathbb{P}^{2}}=\mathcal{O}_{\mathbb{P}^{14} \times \mathbb{P}^{2}}(-15,-3)
$$

it will take a little longer for the fiber power to have positive dualizing sheaf, but it happens: if we let $Y$ be the $15^{\text {th }}$ fiber power of $X$ with itself over $B$, we see that $Y$ is a complete intersection in $\mathbb{P}^{14} \times\left(\mathbb{P}^{2}\right)^{15}$ of 15 hypersurfaces, of multidegrees $(1,4,0, \ldots, 0),(1,0,4,0, \ldots, 0), \ldots,(1,0, \ldots, 0,4)$; and accordingly

$$
\omega_{Y} \cong \mathcal{O}_{Y}(0,1, \ldots, 1)
$$

which is big - in fact, it is very ample.

But $Y$ itself cannot possibly be of general type, for $X$ and likewise all its fiber powers are acted upon by $P G L_{3}$, and the general orbits of these actions are iso- 
morphic to $P G L_{3}$. In particular, the fiber powers will have copies of $P G L_{3}$ passing through a general point and so cannot have any sections of any power of the canonical bundle.

The conclusion is that the situation must be exactly the opposite of that in the case of the pencil: namely, the singularities of the fiber powers $Y$ must not be canonical; and indeed they must impose so many adjoint conditions that none of the (many) sections of powers of $\omega_{Y}$ can extend to regular sections of the canonical bundle of a desingularization.

This shows how important to our proof in the case of curves is the reduction to the case of families with only normal crossing singularities. In fact, everything about the argument except this reduction holds as well in the higher-dimensional case. Explicitly, suppose we are given a family $X \rightarrow B$ whose general fiber is of general type; for simplicity suppose that the spaces $X$ and $B$ are smooth and that the fiber dimension of the map is constant (so that in particular the fiber powers are local complete intersections and therefore have locally free dualizing sheaves). Suppose also that the family has maximal variation of moduli, in the sense of [Ko]. Then we have

i. The relative dualizing sheaf $\omega_{X / B}$ is big (cf. [Ko]).

ii. By our Lemma 3.1, it follows that for $n \gg 0$ the dualizing sheaf of $X_{B}^{n}$ is big.

iii. If the fibers of $X \rightarrow B$ have normal crossing singularities, then the singularities of the fiber powers are canonical (cf. $[\mathrm{V}])$.

iv. Moreover, if in the above setting a finite group $G$ acts simultaneously on $X$ and $B$, then the adjoint conditions imposed by the singularities of the quotient of $X_{B}^{n}$ by the diagonal action of $G$ will again be bounded independently of $n$, by our Corollary 4.2. In particular $X_{B}^{n} / G$ will be of general type for $n \gg 0$.

Thus we see that all the ingredients of a proof in the higher-dimensional case are in place, except for the analog of semistable reduction. In particular, the conjecture would presumably follow from the existence of a compact moduli space for a class of varieties including the general fiber of $X \rightarrow B$ and whose singularities were no worse than normal crossings (or more generally such that the singularities of the fiber powers of their deformations were canonical).

In the remainder of this section, we will discuss two results that would follow from Conjecture $\mathrm{H}$ above, one general and geometric and the other special and arithmetic.

6.2. Geometric consequences of the higher-dimensional conjecture. Recall that, according to the Geometric Lang Conjecture, if $X$ is any variety, then the union of all (positive-dimensional) subvarieties of $X$ not of general type is again a subvariety, which we will call the Langian exceptional locus of $X$ and denote $\Xi_{X} \subset X$. Lang has conjectured further that $\Xi_{X}$ is the Zariski closure of the union of all images of rational and abelian varieties under non-constant maps to $X$, and that it is the smallest subvariety of $X$ satisfying the statement of Conjecture B (the Strong Lang Conjecture) above. Note one property of the Langian locus that follows from any of these characterizations: if $Y \subset X$ is any subvariety of $X$ and $\Xi_{X} \subset Y$, then $\Xi_{X}=\Xi_{Y}$.

Assuming the truth of the Geometric Lang Conjecture, a natural question to ask is how the subvarieties $\Xi$ vary with parameters - that is, if we are given a family $X \rightarrow B$ of varieties of general type, what can we say about the exceptional subvarieties $\Xi_{b}=\Xi_{X_{b}}$ of the fibers? The answer is expressed in the following 
Theorem 6.1. Assuming the Geometric Lang Conjecture and Conjecture $(H)$ above, there is a number $D(d, k)$ such that for all projective varieties $X$ of degree $d$ or less and dimension $k$ or less, the total degree of the Langian exceptional locus is

$$
\operatorname{deg}\left(\Xi_{X}\right) \leq D(d, k) .
$$

Here by the total degree of a variety we mean the sum of the degrees of its irreducible components; this differs from the ordinary notion of degree precisely when the variety does not have pure dimension.

Before proceeding with the proof of Theorem 6.1, we mention a few consequences of this boundedness statement. In perhaps its simplest application, for example, it would say that for any $d \geq 5$ there is an upper bound $L(d)$ on the degree of a curve of geometric genus 0 or 1 lying on a smooth surface $S$ of degree $d$ in $\mathbb{P}^{3}$, independent of the particular surface $S$. (This is actually a consequence of the more general theorem announced by Lu and Miyaoka in [LM].) Likewise, it would say that for any $d \geq 6$ there is an upper bound on the degree of any surface not of general type lying on a smooth threefold $X$ of degree $d$ in $\mathbb{P}^{4}$, and also on the degree of any rational or elliptic curve lying on such a threefold but not contained in any such surface, again independent of the particular hypersurface. Both of these latter statements are completely unknown.

Proof. We will use an induction on the dimension $k$. We start with a sequence of reductions. First of all, since the Hilbert scheme parametrizing subvarieties of projective space of bounded degree and dimension is of finite type, and there exists a universal family over it, it will suffice to prove that, given any morphism $f: X \rightarrow B$ of projective varieties, the degrees of the Langian exceptional loci $\Xi_{X_{b}}$ of the fibers are bounded. Secondly, by the Noetherian property of the Zariski topology, it will suffice to show that in this circumstance there is a non-empty open subset $U \subset B$ such that the degrees of the loci $\Xi_{X_{b}}$ for $B \in U$ are bounded. Third, we may by pulling back assume that the base $B$ is smooth; and fourth, replacing $X$ by its normalization we may assume that the general fiber of $f$ is smooth. Finally, since we are only concerned with an open subset of $B$, we can assume the morphism $f$ is smooth.

Now, by upper-semicontinuity of the dimension of the space of global sections of a family of coherent sheaves applied to the powers of the relative dualizing sheaf, if $f: X \rightarrow B$ is a smooth morphism, then the locus of points $b \in B$ over which the fiber $X_{b}$ is of general type is closed. If in our present circumstance this locus is a proper subvariety $Z \subset B$, we are trivially done, since then we may restrict to the complement $U=B \backslash Z$, and $\Xi_{X_{b}}=X_{b}$ for all $b$ in this open set. Otherwise, all the fibers of $f$ are of general type.

We may thus apply the Conjecture $\mathrm{H}$ to our family, to conclude that for $n$ sufficiently large the fiber power $X_{B}^{n}$ admits a dominant rational map $h$ to a variety $W$ of general type. Let $Z \subset X_{B}^{n}$ be the union of two subvarieties of $X_{B}^{n}$ : the inverse image in $X_{B}^{n}$ of the Langian exceptional locus of $W$; and the locus of positivedimensional fibers of the map $f \times h: X_{B}^{n} \rightarrow B \times W$ - that is, the union of the loci of positive-dimensional fibers of the restrictions of $h$ to the fibers of $f$. $Z$ is then a proper subvariety of $X_{B}^{n}$ that, for every point $u$ in $X_{B}^{n-1}$, contains the Langian exceptional locus of the fiber of $X_{B}^{n}$ over $u$.

Consider now the projection map $\pi_{n}: X_{B}^{n} \rightarrow X_{B}^{n-1}$ and its restriction to $Z$; note in particular that for any choice of projective embedding of $X_{B}^{n}$, the degrees 
of the fibers of $Z$ over $X_{B}^{n-1}$ are bounded. Let $Z_{n-1} \subset X_{B}^{n-1}$ be the locus of points $u \in X_{B}^{n-1}$ such that $Z$ contains the whole fiber of $X_{B}^{n}$ over $u$, and let $U_{n-1} \subset X_{B}^{n-1}$ be its complement; let $U_{0}$ be the image of $U_{n-1}$ in $B$, which contains a non-empty open subset $U \subset B$. For every point $b \in U$, there is a point $u \in X_{B}^{n-1}$ lying over $b$ such that $Z$ does not contain the fiber of $X_{B}^{n}$ over $u$; thus the Langian exceptional loci of the fibers of $X$ over $b \in U$ are the Langian exceptional loci of the fibers of $Z$ over points of $U_{n-1}$. But these fibers form a family of bounded degree and dimension $k-1$ or less; so by induction the degrees of their Langian exceptional loci are bounded.

6.3. An arithmetic consequence of the higher-dimensional conjecture. In contrast to the general nature of Theorem 6.1 , this will be a very special application of Conjecture H; indeed, we will assume only the Lang conjectures and Conjecture $\mathrm{H}$ for the symmetric fiber squares of families of curves.

What makes it possible to deduce consequences of the conjectures in this case is simply that we have some knowledge of the Langian exceptional loci of symmetric squares of curves. Specifically, if $C$ is a hyperelliptic curve, the symmetric square of $C$ will contain a rational curve, namely the fibers of the degree 2 map from $C$ to the projective line. Similarly, if $C$ is bielliptic - that is, admits a map $\pi$ of degree 2 to an elliptic curve $E$ - then the fibers of $\pi$ give an elliptic curve in the symmetric square $C^{(2)}$ of $C$. Less obviously, we have a converse to these observations: if $C$ is any curve that is assumed to be neither hyperelliptic nor bielliptic, then by $[\mathrm{AH}]$ the symmetric square of $C$ will contain no rational or elliptic curves; and the Langian exceptional locus of $C^{(2)}$ will thus be empty.

Assuming the Weak Lang Conjecture, if $X \rightarrow B$ is a family of curves with no hyperelliptic or bielliptic fibers, then the number of rational points on the fibers of the symmetric fiber square will be bounded. Thus, we may deduce from the Weak Lang Conjecture that for every number field $K$ and any non-hyperelliptic, non-bielliptic curve $C$ of genus $g$ defined over $K$, there is a uniform bound on the total number of points of $C$ whose coordinates are quadratic over $K$, independent of the particular curve $C$. Similarly, from the Strong Lang Conjecture we could deduce the existence of a bound $\mathrm{N}_{q}(g)$, independent of $K$, that applies with finitely many exceptions for any $K$. We state this formally:

Theorem 6.2. Assuming the Weak Lang Conjecture and Conjecture $H$ for families of symmetric squares of curves, there exists for every integer $g$ and number field $K$ a number $\mathrm{B}_{q}(g, K)$ such that no non-hyperelliptic, non-bielliptic curve $C$ of genus $g$ defined over $K$ has more than $\mathrm{B}_{q}(g, K)$ points whose coordinates are quadratic over $K$. If we assume in addition the Strong Lang Conjecture, there exists for every integer $g$ a number $\mathrm{N}_{q}(g)$ such that for any number field $K$ there are only finitely many non-hyperelliptic, non-bielliptic curves of genus $g$ defined over $K$ that have more than $N_{Q}(g)$ points whose coordinates are quadratic over $K$.

\section{REFERENCES}

[Ab1] D.Abramovich. On the number of stably integral points on an elliptic curve. preprint. [Ab2] D.Abramovich. Uniformité des points rationnels des courbes algébriques sur les extensions quadratiques et cubiques. preprint. 
[AH] D.Abramovich, J.Harris. Abelian varieties and curves in $W_{d}(C)$. Compositio Math. 78 (1991) p. 227-238. MR 92c: 14022

[Ar] S.Ju.Arakelov. Families of algebraic curves with fixed degeneracies. Izv. Akad. Nauk. SSSR, Ser. Math 35 (1971); English translation Math USSR Izv. 5 (1971) p. 1277-1302. MR 48:298

[ACGH] E.Arbarello, M.Cornalba, P.Griffiths, J.Harris. Geometry of Algebraic Curves, Volume 1. Springer-Verlag, NY. MR 86h:14019

[BM] E. Bierstone, P. Milman. A simple constructive proof of canonical resolution of singularities. In: Effective Methods in Algebraic Geometry, Progress in Math. vol. 94, Birkhauser Boston 1991, p. 11-30. MR 92h:32053

[B] F.Bogomolov. Families of curves on surfaces of general type. Dokl. AN SSSR 236 (6) (1977) p. 1294-1297 (English: Sov. Math. Doklady 1041-1044). MR 56:15655

$[\mathrm{CHM}]$ L.Caporaso, J.Harris, B.Mazur. How many rational points can a curve have? Proceedings of the Texel Conference, Progress in Math. vol. 129, Birkhauser Boston, 1995, p. 13-31. CMP 96:04

[CHM1] L.Caporaso, J.Harris, B.Mazur. Uniformity of rational points. Preliminary version of this paper, available by anonymous ftp from math.harvard.edu.

[SGA] M.Demazure Exposé IV: Topologies et Faisceaux 6.5, 6.5. In: SGA 3 (Schemas en Groupes I), Springer Lecture Notes in Mathematics 151 (1970) MR 43:223a

[E] L.Ein. Subvarieties of generic complete intersections, II. Math. Ann. (289), pp 465-471. MR 92h:14002

[El] R.Elkik, Singularités rationnelles et deformations., Invent. Math. 47, (1978) p. 139-147. MR 80c: 14004

[F] G.Faltings. The general case of S. Lang's Conjecture. Barsotti Symposium in Algebraic Geometry, Perspectives in Mathematics, Academic Press, Inc. 1994 p.175-182. MR 95m:11061

[EGA] A.Grothendieck. Eléments de Géométrie Algébrique, Chap. 3 Sect. 6.3. (1961). MR 29:1209

[H] R.Hartshorne. Ample subvarieties of algebraic varieties. Lecture Notes in Math. 156. Springer Verlag (1970). MR 44:211

[Ha] B.Hassett. Correlation for surfaces of general type. preprint.

[Hi] H. Hironaka. Idealistic exponents of singularity. In: Algebraic Geometry, J.J.Sylvester Symposium, Johns Hopkins Univ., Baltimore, Md., 1976, Johns Hopkins Univ. Press, Baltimore, Md., 1977, p. 52-125. MR 58:16661

[Ko] J.Kollár. Subadditivity of the Kodaira dimension: fibers of general type. Adv. Stud. in Pure Math. 10 (1987) p. 361-398. MR 89i:14029

[Ko1] J.Kollár. Projectivity of complete moduli. J. Diff. Geom 32 (1990) 235-268. MR 92e: 14008

[L] S.Lang. Hyperbolic and diophantine analysis. Bull. Amer. Math. Soc. 14 , No. 2 (1986) p. 159-205. MR 87h:32051

[Lo] E.Looijenga. Smooth Deligne-Mumford compactifications by means of Prym levels structires. J.AlgGeom. 3 (1992) p.283-293.

[LM] S.Lu, M. Miyaoka. preprint.

$[\mathrm{Mu}] \quad$ D.Mumford. Stability of projective varieties. L'Enseignement Mathématique 23 (1977) p. 39-110. MR 56:8568

[GIT] D.Mumford, J.Fogarty. Geometric Invariant Theory. Springer Verlag (1982). MR 86a: 14006

[Po1] H.Popp. Modulräume algebraischer Mannigfaltigkeiten. Classification of Algebraic Varieties and Compact Complex Manifolds, Springer Lecture Notes 412, 1974. MR 50:13029

[Po2] H.Popp. Moduli Theory and Classification Theory of Algebraic Varieties. Springer Lecture Notes 620, 1977. MR 57:6024

[R] M.Reid. Canonical threefolds. Géométrie Algébrique, Angers 1979 Sijthoff and Nordhoff (1980) p. 273-310. MR 82i: 14025

[V] E.Viehweg. Weak positivity and the additivity of the Kodaira dimension for certain fibre spaces. Adv. Stud. in Pure Math. 1 (1983) p. 329-353.

[V1] E.Viehweg. Canonical divisors and the additivity of the Kodaira dimension for morphisms of relative dimension one. Compositio Math. 35, Fasc 2 (1977) p. 197-223. 
[V2] E.Viehweg. Rational singularities of higher dimensional schemes. Proc. AMS. 63 n.1 (1977) p.6-8.

[Vo] P.Vojta Diophantine Approximations and Value Distribution Theory. Springer Lecture Notes in Mathematics 1239 (1987) MR 91k:11049

Department of Mathematics, Harvard University, 1 Oxford St., Cambridge, MasSACHUSETTS 02138

E-mail address: caporaso@zariski.harvard.edu

E-mail address: harris@zariski.harvard.edu

E-mail address: mazur@zariski.harvard.edu 